\title{
Energy-based reformulated Craig-Bampton method for multiple flexural subsystems connected at a junction with low impedance mismatch
}

\author{
Laurent Maxit ${ }^{1}$ and Oriol Guasch ${ }^{2}$ \\ 1. INSA-Lyon, Laboratoire Vibrations-Acoustique (LVA), 25 bis, av. Jean Capelle, F-69621, \\ Villeurbanne Cedex, France. \\ e-mail: laurent.maxit@insa-lyon.fr (corresponding author) \\ 2. GTM - Grup de recerca en Tecnologies Mèdia, La Salle, Universitat Ramon Llull, C/ Quatre \\ Camins 2, 08022 Barcelona, Catalonia, Spain. \\ e-mail: oriol.guasch@salle.url.edu
}

\begin{abstract}
:
Whereas the coupling between modes of two different subsystems is well-resolved in vibroacoustic energy-based methods, the situation becomes more intricate when several subsystems get connected at a common junction. In statistical energy analysis (SEA), the modal formulation is replaced by the travelling wave approach to solve the problem. However, this is not a viable option for other energy-based methods, like the statistical modal energy distribution analysis (SmEdA), and a modal coupling scheme is required for them. If there is a strong impedance mismatch between the multiple connected subsystems, the displacement-stress dual formulation offers a proper way to address the situation. Yet, the latter fails if all involved subsystems have similar stiffness. In this work, the feasibility of the Craig-Bampton (CB) method to address such circumstance is explored. It is shown that the original $\mathrm{CB}$ technique does not fulfill the modal coupling assumptions of energybased methods, so it is suggested to reformulate it to partially mitigate the problem. Numerical tests on a benchmark problem are carried out to validate the proposal. The benchmark consists of a floor coupled with two walls at right angle, and it is analyzed for different impedance mismatch conditions.
\end{abstract}

Key words:

Statistical energy analysis, modal formulation, component modal synthesis, power flow, mid-frequency modelling, plate assembly, Craig-Bampton method 


\section{Nomenclature}

The symbols used in the main text are described in this section.

$E_{k, \varphi \varphi}^{\alpha} \quad$ kinetic energy related the normal modes of subsystem $\alpha$ for an unit harmonic excitation

$E_{k, \varphi \psi}^{\alpha} \quad$ kinetic energy arising from the cross interaction between the normal and the characteristic constraint modes for an unit harmonic excitation

$E_{k, \psi \psi}^{\alpha} \quad$ kinetic energy of subsystem $\alpha$ related to the characteristic constraint modes for an unit harmonic excitation

$E_{p, \varphi \varphi}^{\alpha} \quad$ strain energy related the normal modes of subsystem $\alpha$ for an unit harmonic excitation

$E_{p, \psi \psi}^{\alpha} \quad$ strain energy of subsystem $\alpha$ related to the characteristic constraint modes for an unit harmonic excitation

f finite element external force vector of the global system

$\mathbf{f}_{B} \quad$ external force vector related to the boundary degrees of freedom

$\mathbf{f}_{g}^{C} \quad$ force vector related to the coupling modes

$\mathbf{f}_{g-C C}$ generalized force vector related to the subsystem normal modes and the characteristic constraint mode

$\mathbf{f}_{g}^{N R+I} \quad$ force vector related to the non-resonant (NR) normal modes, plus the constraint interface (I) modes (NR+I modes)

$\mathbf{f}_{g}^{R} \quad$ force vector related to the resonant normal modes

$\mathbf{f}_{I} \quad$ external force vector related to the interior degrees of freedom

$\mathbf{f}_{I}^{1} \quad$ external force vector related to the interior degrees of freedom applied on subsystem 1

I identity matrix

$\mathbf{k}_{B B} \quad$ stiffness matrix related to the boundary degrees of freedom

$\mathbf{k}_{I B} \quad$ coupling stiffness matrix between the interior degrees of freedom and the boundary degrees of freedom

$\mathbf{k}_{I I} \quad$ stiffness matrix related to the interior degrees of freedom

$\mathbf{k}_{\psi \psi}$

$\overline{\mathbf{k}}_{\psi \psi}^{\alpha}$

stiffness matrix related to the constraint modes

stiffness matrix of subsystem $\alpha$ related to the characteristic constraint modes

$\mathbf{K}$ finite element stiffness matrix of the considered system

$\mathbf{K}_{g-C C} \quad$ stiffness matrix related to the subsystem normal modes and the characteristic constraint mode

$\mathbf{K}_{g}^{N R+I} \quad$ stiffness matrix related to the non-resonant (NR) normal modes, plus the constraint interface (I) modes (NR+I modes)

$\mathbf{m}_{B B} \quad$ mass matrix related to the boundary degrees of freedom

$\mathbf{m}_{I B} \quad$ coupling mass matrix between the interior degrees of freedom and the boundary degrees of freedom 


\begin{tabular}{|c|c|}
\hline $\mathbf{m}_{I I}$ & mass matrix related to the interior degrees of freedom \\
\hline $\mathbf{m}_{\phi \phi}$ & mass matrix related to the normal modes \\
\hline $\mathbf{m}_{\varphi \varphi}^{\alpha-N R}$ & mass matrix related to the non-resonant normal modes of subsystem $\alpha$ \\
\hline$\overline{\mathbf{m}}_{\phi \psi}^{\alpha}$ & $\begin{array}{l}\text { coupling mass matrix between the subsystem modes of subsystem } \alpha \text { and the } \\
\text { characteristic constraint modes }\end{array}$ \\
\hline $\mathbf{m}_{\varphi \psi}^{\alpha-N R}$ & $\begin{array}{l}\text { coupling mass matrix between to the non-resonant normal modes of subsystem } \\
\alpha \text { and the constraint modes }\end{array}$ \\
\hline $\mathbf{m}_{\varphi \psi}^{\alpha-R}$ & $\begin{array}{l}\text { coupling mass matrix between to the resonant normal modes of subsystem } \alpha \text { and } \\
\text { the constraint modes }\end{array}$ \\
\hline $\mathbf{m}_{\varphi \varphi}^{\alpha-R}$ & mass matrix related to the resonant normal modes of subsystem $\alpha$ \\
\hline $\mathbf{m}_{\phi \psi}$ & coupling mass matrix between the normal modes and the constraint modes \\
\hline $\mathbf{m}_{\psi \psi}$ & mass matrix related to the constraint modes \\
\hline$\overline{\mathbf{m}}_{\psi \psi}^{\alpha}$ & mass matrix of subsystem $\alpha$ related to the characteristic constraint modes \\
\hline M & finite element mass matrix of the considered system \\
\hline $\mathbf{M}_{g}^{C}$ & mass matrix related to the coupling modes \\
\hline $\mathbf{M}_{g-C C}$ & $\begin{array}{l}\text { global mass matrix related to the subsystem normal modes and the characteristic } \\
\text { constraint mode }\end{array}$ \\
\hline $\mathbf{M}_{g}^{N R+I}$ & $\begin{array}{l}\text { global mass matrix related to the non-resonant (NR) normal modes, plus the } \\
\text { constraint interface (I) modes (NR+I modes) }\end{array}$ \\
\hline $\mathbf{M}_{g}^{R}$ & global mass matrix related to the resonant normal modes \\
\hline $\mathbf{M}_{g}^{R / C}$ & $\begin{array}{l}\text { global coupling mass matrix between the resonant normal modes and the } \\
\text { coupling modes }\end{array}$ \\
\hline $\mathbf{M}_{g}^{R / N R+I}$ & $\begin{array}{l}\text { global coupling mass matrix between the resonant }(\mathrm{R}) \text { normal modes and the } \\
\text { non-resonant }(\mathrm{NR}) \text { normal modes, plus the constraint interface (I) modes (NR+I } \\
\text { modes) }\end{array}$ \\
\hline $\mathbf{q}_{C C}$ & amplitude vector of the characteristic constraint mode \\
\hline $\mathbf{q}_{g}^{C}$ & amplitude vectors related to the coupling modes \\
\hline $\mathbf{q}_{g-C C}$ & $\begin{array}{l}\text { global amplitude vector related to the subsystem normal modes and the } \\
\text { characteristic constraint mode }\end{array}$ \\
\hline $\mathbf{q}_{g}^{N R+I}$ & $\begin{array}{l}\text { global amplitude vectors related to the non-resonant (NR) normal modes, plus } \\
\text { the constraint interface (I) modes (NR+I modes) }\end{array}$ \\
\hline $\mathbf{q}_{g}^{R}$ & global amplitude vectors related to the resonant normal modes \\
\hline $\mathbf{q}_{\phi}$ & amplitude vector of the normal modes of the subsystem with fixed interface \\
\hline $\mathbf{q}_{\varphi}^{\alpha-N R}$ & amplitude vectors related to the non-resonant normal modes of subsystem $\alpha$ \\
\hline $\mathbf{q}_{\varphi}^{\alpha-R}$ & amplitude vectors related to the resonant normal modes of subsystem $\alpha$ \\
\hline $\mathbf{q}_{\psi}$ & amplitude vector of the constraint modes \\
\hline$S_{F F}$ & power spectral density of the force signal \\
\hline $\mathbf{u}$ & displacement vector of the global system \\
\hline
\end{tabular}




\begin{tabular}{|c|c|}
\hline $\mathbf{u}_{B}$ & displacement vector related to the boundary degrees of freedom \\
\hline $\mathbf{u}_{I}$ & displacement vector related to the interior degrees of freedom \\
\hline$\Gamma$ & matrix containing the eigenvectors related to the $N_{C}$ first coupling modes \\
\hline$\varphi i$ & eigenvector related to the $i$-th eigenvalue $\lambda_{C}^{i}$ of the generalized eigenvalue \\
\hline & $\begin{array}{l}\text { problem defined by the mass and stiffness matrices of the non-resonant normal } \\
\text { modes, plus the constraint interface modes (i.e. eigenvector related to the } i \text {-th } \\
\text { coupling mode) }\end{array}$ \\
\hline & damping loss factor \\
\hline$\lambda^{i}$ & eigenvalue of the $i$-th normal modes of the subsystem with fixed interface \\
\hline$\lambda_{C}^{i}$ & eigenvalue of the $i$-th coupling modes \\
\hline$\lambda_{C C}^{i}$ & eigenvalue of the $i$-th characteristic constraint modes \\
\hline$\Lambda^{a}$ & eigenvalue diagonal matrix of subsystem $\alpha \in\{1,2,3,4\}$ \\
\hline$\Lambda^{C}$ & eigenvalue diagonal matrix related to the coupling modes \\
\hline$\Lambda_{C C}$ & eigenvalue diagonal matrix related to the characteristic constraint mode \\
\hline$\Lambda_{g}^{R}$ & eigenvalue diagonal matrix related to the resonant normal modes \\
\hline$\Lambda_{\phi \phi}$ & $\begin{array}{l}\text { eigenvalue diagonal matrix related to the normal modes of the subsystem with } \\
\text { fixed interface }\end{array}$ \\
\hline$\Lambda_{\varphi \varphi}^{\alpha-N R}$ & $\begin{array}{l}\text { eigenvalue diagonal matrix related to the non-resonant normal modes of } \\
\text { subsystem } \alpha\end{array}$ \\
\hline$\Lambda_{\varphi \varphi}^{\alpha-R}$ & $\begin{array}{l}\text { eigenvalue diagonal matrix related to the resonant normal modes of subsystem } \\
\alpha\end{array}$ \\
\hline & $\begin{array}{l}\text { matrix containing the eigenvectors related to the } N_{C C} \text { first characteristic } \\
\text { constraint modes }\end{array}$ \\
\hline$e^{i}$ & eigenvector related to the $i$-th eigenvalue $\lambda_{C C}^{i}$ of the generalized eigenvalue \\
\hline & $\begin{array}{l}\text { problem defined by the mass and stiffness matrices related to the constraint } \\
\text { modes (i.e. eigenvector related to the } i \text { th Characteristic Constraint (CC) mode) }\end{array}$ \\
\hline${ }_{I I}^{i}$ & $\begin{array}{l}\text { eigenvector related to the } i \text {-th eigenvalue } \lambda^{i} \text { of the generalized eigenvalue } \\
\text { problem defined by the mass and stiffness matrices related to the interior degrees } \\
\text { of freedom (i.e. eigenvector related the } i \text {-th normal mode of the considered } \\
\text { subsystem with fixed interface) }\end{array}$ \\
\hline$\Phi$ & matrix containing the eigenvectors related to the $N_{I I}$ first normal modes of the \\
\hline$\psi$ & $\begin{array}{l}\text { nsidered subsystem with fixed interface } \\
\text { atrix containing the static shapes related to the interior and boundary degrees } \\
\text { e. constraint modes) }\end{array}$ \\
\hline$\psi_{1}$ & atrix containing the static shapes related to the interior degrees of freedom \\
\hline & lar frequency \\
\hline
\end{tabular}




\section{Introduction}

It is well-known that statistical energy analysis (SEA) admits two distinct formulations [13]. The first one arises from statistical considerations on how normal modes in a built-up structure interchange energy and is referred to as the modal approach to SEA. The second one grounds on statistical developments of disordered travelling waves propagating in a structure and is named the wave approach to SEA. The advantage of such a twofold formulation is that when difficulties are encountered when applying the modal one, those can be often overcome by switching to the wave one, and vice versa.

An example of such a type concerns the connection of several subsystems at a junction. In SEA a subsystem is identified with a group of resonant modes. Under certain conditions, which we denote as the modal coupling assumptions (MCAs) (to be specified below), the modal approach establishes the power balance equations between two distinct subsystems. However, it is not clear at all whether the MCAs generalize to multiple subsystems connected at a junction. SEA circumvents the problem by resorting to the wave approach to characterize that situation (see e.g., [4-6]). Yet in a recent work [7], it was shown that it was also possible to establish a modal scheme satisfying the MCAs, whenever there was a strong impedance mismatch between the subsystems sharing the joint. The dual modal stress-displacement formulation was used for that purpose [8-12]. The current work is to be viewed as a follow-up of [7], where we now address the case of all connected subsystems having similar stiffness i.e, when there is a low impedance mismatch at the joint.

One might argue that such a problem is only of theoretical interest to SEA, given that in practice one could always avoid it by switching to the wave formulation. Even if that was the case, which is still believe interesting per se, there are other energy-based methods where there is a need for a multiple connection scheme that complies with the MCAs. The strong hypotheses underlying SEA ([1,2]) limit the theory to the high frequency range, so many methods have been proposed to extend its applicability to lower frequencies and face the so-called mid frequency problem. On the one hand, the wave approach to SEA can be derived from more general ray-tracing techniques under the assumption of diffusivity (see e.g., $[13,14]$ ). On the other hand, in what concerns modal extensions to SEA, several methods have been proposed. For instance, in the energy 
distribution analysis (EDA) [15,16] energy transmission is characterized by means of energy influence coefficients, which are related to structural global modes. In the Asymptotical Scaled Modal Analysis (ASMA) method [17-20] a scaling procedure is established to work with a reduced model that represents the average behavior of the original system. Lastly, the statistical modal energy distribution analysis (SmEdA) method [21-24] proposes power balance equations between individual resonant modes belonging to different subsystems, rather than between subsystems themselves, as in SEA. In fact, the latter can be obtained as a limit case of SmEdA. It is to be noted that SmEdA fulfils the MCAs, like SEA, but has no wave approach analogue, so a modal coupling scheme for multiple connected subsystems it is not only an issue of academic interest for SmEdA, but a subject of very practical interest.

As said before, it was concluded in [7] that the DMF works well when there is a strong impedance mismatch between subsystems at the junction, but it is not a valid modal approach for low impedance mismatch values. In such situation each subsystem perceives the feedback of the other ones at the junction and may be well approximated as having clamped boundary conditions there. It was consequently suggested in [7] that one could resort to the celebrated Craig-Bampton (CB) formulation $[25,26]$ instead of the DMF. In the $\mathrm{CB}$ method, the fixed-interface component modes (i.e., blocked modes) are computed for each subsystem and complemented with the so-called constraint modes. Thereby, the fixed-interface component modes could be - a priori - well adapted to represent the global behavior of coupled subsystems.

The organization of the paper is as follows. Section 2 reviews the MCAs and give a brief outline of the CB formulation. Section 3 presents a benchmark problem consisting of a floor and two walls at right angle, which was used to illustrate the developments in [7]. We resort to it once more to allow comparison with the formulations in this work. The modal equations for the benchmark problem are derived in section 4 for the original $\mathrm{CB}$ method, followed by numerical simulations and a discussion. It is shown that, unfortunately, the standard CB does not comply with the MCAs, so an alternative CB formulation is proposed and tested in section 5, to improve the results. Finally, conclusions are drawn in section 6 . 


\section{Theoretical framework}

\subsection{Modal coupling assumptions}

To make the present paper self-contained, let us reproduce the set of modal coupling assumptions (MCAs) listed in [7] that, for energy-based methods, describe the interaction between modes, or groups of modes, belonging to two different subsystems. The MCAs, state that (see also $[1,2])$ :

- the interaction only involves the modes of the uncoupled subsystems,

- the dynamics of a subsystem mode are those of an oscillator (mass-springdamper system),

- the coupling between modes in different subsystems is conservative and happens through mass, stiffness and/or gyroscopic elements,

- modes within a subsystem are uncoupled (i.e., the modes conform an orthogonal set),

- the resonant modes of each subsystem account for all energy interchanged with other subsystems.

\subsection{Fundamentals of the Craig-Bampton method}

To facilitate the comprehension of the forthcoming sections, let us next present an outline of the most meaningful results of the original Craig-Bampton method [25,26]. To begin with, consider a nonspecific finite element (FEM) model of a single subsystem, its equation of motion being given by

$$
\mathbf{M} \ddot{\mathbf{u}}+\mathbf{K u}=\mathbf{f},
$$

where $\mathbf{u}$ stands for the nodal displacement vector and $\mathbf{f}$ for the external applied force vector. As usual, a dot symbol designates time derivative. M stands for the FEM mass matrix and $\mathbf{K}$ for the stiffness one.

Let us partition the displacements and applied forces into

$$
\mathbf{u}=\left[\begin{array}{l}
\mathbf{u}_{I} \\
\mathbf{u}_{B}
\end{array}\right], \quad \mathbf{f}=\left[\begin{array}{l}
\mathbf{f}_{I} \\
\mathbf{f}_{B}
\end{array}\right],
$$

where the vector $\mathbf{u}_{I}$ contains the displacements of the interior degrees of freedom (d.o.f.s) of the subsystem and $\mathbf{u}_{B}$ encompasses the displacements of the boundary d.o.f.s. 
Analogously, $\mathbf{f}_{I}$ stands for the forces applied on the interior d.o.f.s and $\mathbf{f}_{B}$ for those at the boundary.

The mass and stiffness matrices in Eq. (1) are partitioned accordingly as

$$
\mathbf{M}=\left[\begin{array}{ll}
\mathbf{m}_{I I} & \mathbf{m}_{I B} \\
\mathbf{m}_{B I} & \mathbf{m}_{B B}
\end{array}\right] \text { and } \mathbf{K}=\left[\begin{array}{ll}
\mathbf{k}_{I I} & \mathbf{k}_{I B} \\
\mathbf{k}_{B I} & \mathbf{k}_{B B}
\end{array}\right] \text {. }
$$

The Craig-Bampton method allows one to rewrite Eq. (1) with the splitting in Eqs. (2)-(3) using two different sets of modes, namely,

(a) The normal modes with fixed-interface, $\Phi$ :

These modes are calculated with all boundary d.o.f.s held fixed (i.e. $\mathbf{u}_{B}=0$ ). The $i$-th mode is represented by the eigenvector $\varphi_{\text {II }}^{i}$, with eigenvalue $\lambda^{i}$, which is obtained from the generalized eigenvalue problem

$$
\left[\mathbf{k}_{I I}-\lambda^{i} \mathbf{m}_{I I}\right] \boldsymbol{\varphi}_{I I}^{i}=0, \forall i \in\left\{1,2, . ., N_{I I}\right\} .
$$

$N_{I I}$ is the total number of modes retained to represent the subsystem. The eigenvectors $\boldsymbol{\varphi}_{I I}^{i}$ can be ranged as the columns of a matrix $\boldsymbol{\varphi}_{I I}=\left[\begin{array}{lll}\boldsymbol{\varphi}_{I I}^{1} & \boldsymbol{\varphi}_{I I}^{2} \ldots \varphi_{I I}^{N_{I I}}\end{array}\right]$ to finally build the normal mode matrix

$$
\Phi=\left[\begin{array}{c}
\boldsymbol{\varphi}_{I I} \\
0
\end{array}\right]
$$

(b) The constraint modes, $\psi$ :

A constraint mode is defined as the static deflection induced in the subsystem when applying a unit displacement at one boundary d.o.f while keeping all remaining boundary d.o.f.s fixed. The constraint mode matrix is given by (see $[25,26])$

$$
\boldsymbol{\psi}=\left[\begin{array}{l}
\boldsymbol{\psi}_{I B} \\
\mathbf{I}_{B B}
\end{array}\right]=\left[\begin{array}{c}
-\left[\mathbf{k}_{I I}\right]^{-1} \mathbf{k}_{I B} \\
\mathbf{I}_{B B}
\end{array}\right] .
$$

The constraint modes provide a complete set of deformation shapes associated with the motion of the boundary d.o.f.s. 
The two type of modes defined by Eqs. (5-6) can be concatenated into a matrix $\mathbf{B}=\left[\begin{array}{ll}\Phi & \psi\end{array}\right]$ that can be used to transform the displacement vector $\mathbf{u}$ to modal coordinates $\mathbf{q}=\left[\begin{array}{ll}\mathbf{q}_{\phi} & \mathbf{q}_{\psi}\end{array}\right]^{T}$,

$$
\mathbf{u}=\mathbf{B q} .
$$

Introducing Eq. (7) in Eq. (1) and projecting the equations on the basis $\mathbf{B}$, provides

$$
\left[\begin{array}{cc}
\mathbf{m}_{\phi \phi} & \mathbf{m}_{\phi \psi} \\
\mathbf{m}_{\phi \psi}^{T} & \mathbf{m}_{\psi \psi}^{T}
\end{array}\right] \ddot{\mathbf{q}}+\left[\begin{array}{cc}
\boldsymbol{\Lambda}_{\phi \phi} & \mathbf{0}_{\phi \psi} \\
\mathbf{0}_{\phi \psi}^{T} & \mathbf{k}_{\psi \psi}^{T}
\end{array}\right] \mathbf{q}=\left[\begin{array}{c}
\boldsymbol{\varphi}_{I I} \mathbf{f}_{I} \\
\boldsymbol{\Psi}_{I B}{ }^{T} \mathbf{f}_{I}
\end{array}\right]+\left[\begin{array}{c}
\mathbf{0} \\
\mathbf{f}_{B}
\end{array}\right],
$$

with

$$
\begin{gathered}
\mathbf{m}_{\phi \phi}=\boldsymbol{\varphi}_{I I}^{i}{ }^{T} \mathbf{m}_{I I} \boldsymbol{\varphi}_{I I}^{i}, \mathbf{m}_{\psi \psi}=\mathbf{m}_{B B}+\mathbf{m}_{B I} \boldsymbol{\psi}_{I B}+\boldsymbol{\psi}_{I B}{ }^{T} \mathbf{m}_{B I}^{T}+\boldsymbol{\psi}_{I B}{ }^{T} \mathbf{m}_{I I} \boldsymbol{\Psi}_{I B}, \\
\mathbf{m}_{\phi \psi}=\mathbf{m}_{B I} \boldsymbol{\varphi}_{I I}+\boldsymbol{\Psi}_{I B}{ }^{T} \mathbf{m}_{I I} \boldsymbol{\varphi}_{I I}, \\
\mathbf{k}_{\psi \psi}=\mathbf{k}_{B B}+\boldsymbol{\psi}_{I B}{ }^{T} \mathbf{k}_{I B}, \quad \boldsymbol{\Lambda}_{\phi \phi}=\boldsymbol{\varphi}_{I I}{ }^{T} \mathbf{k}_{I I} \boldsymbol{\varphi}_{I I}=\operatorname{diag}\left[\lambda^{i}\right]_{N_{I I} \times N_{I I}}
\end{gathered}
$$

\section{Benchmark problem and sub-structuring}

The developments concerning the $\mathrm{CB}$ approaches in the forthcoming sections will be applied to the same benchmark problem in [7]. This will ease comparison with the results reported in that previous work. The benchmark is depicted in Figure 1 and consists of a structure where four panels share a junction at right angle. The floor is made of panels P1 and $\mathrm{P} 2$ while panels $\mathrm{P} 3$ and $\mathrm{P} 4$ constitute vertical walls. Only flexural motions of the panels are considered to facilitate the analytical developments.

Two test cases corresponding to strong and low impedance mismatches at the junction will be contemplated. In test case \#1 the floor is made of concrete and it is $20 \mathrm{~cm}$ in thickness, the walls being brickwork constructed and $5 \mathrm{~cm}$ in thickness. It was shown in [7] that in such situation the structure could be divided into three subsystems: a single subsystem for the floor (panels P1 + P2) and two more subsystems for each wall (see Figure 2a). The DMF performed well in that case and provided a modal coupling scheme in accordance with the MCAs. This was because there is a strong impedance mismatch between the floor and the walls. However, in a second test case \#2, all panels were assumed to be made of concrete and of $20 \mathrm{~cm}$ in thickness. The DMF failed in that occasion, and it 
was argued in [7] that a sub-structuring scheme in which every panel was identified with a subsystem, having clamped conditions at the junction, would be more appropriate for test case \#2 (see Figure 2b). This is so because all panels having the same stiffness, their motion gets blocked at the junction by the three remaining ones. Our goal in the forthcoming section will be to check whether, as suggested in [7], the CB method could perform better than the DMF for test case \#2, and see if it also works well for test case \#1.

The modes of the four panels needed for the CB developments have been obtained from a FEM model and the SDTool code in MATLAB [27]. The whole benchmark structure has been meshed with 51604 quadrilateral shell elements (52126 nodes). This mesh has been used for the two test cases and fulfils the criterion of six elements per flexural wavelength at $2 \mathrm{kHz}$ for test case \#1. Given that results will be presented for the $1 \mathrm{kHz}$ octave band, the mesh is fine enough to get negligible numerical errors in the two test cases. 134 nodes have been placed at the structure junction with blocked translations in the three directions (only rotation is allowed as we are only considering flexural motion). Besides, clamped boundary conditions have been imposed on the nodes of the outer edges.

The meshes of the subsystems have been directly extracted from the global mesh of the structure and the subsystem modes obtained from the Implicitly Restarted Lanczos Method (eigs command in SDTool, [27]). In what concerns external excitation, a normal

force with unit power spectral density $\left(S_{F F}(f)=1 \mathrm{~N}^{2} / \mathrm{Hz}\right.$ ) has been applied on panel $\mathrm{P} 1$, at the $1 \mathrm{kHz}$ central frequency octave band. The excitation point has coordinates $(0 \mathrm{~m}, 1.6 \mathrm{~m}$, $0.87 \mathrm{~m})$. Besides, the damping loss factor of all panels has been set to $\eta=0.02$ for the two test cases.

\section{Craig-Bampton method for multiple subsystems coupled at a junction}

\subsection{Modal equations for four subsystems coupled at a junction}

The CB developments in section 2.2 can be extended to the four-panel benchmark structure in Figure 1. To assemble the subsystem matrices, displacement continuity and force equilibrium have been imposed at the junction using the Boolean assembly matrix [25,26]. Given that the external excitation is only applied to an internal d.o.f. of subsystem 1, the four-panel $\mathrm{CB}$ modal equations become 


$$
\mathbf{M}_{g} \ddot{\mathbf{q}}_{g}+\mathbf{K}_{g} \mathbf{q}_{g}=\mathbf{f}_{g},
$$

with

$$
\begin{aligned}
& \mathbf{q}_{g}=\left[\begin{array}{c}
\mathbf{q}_{\phi}^{1} \\
\mathbf{q}_{\phi}^{2} \\
\mathbf{q}_{\phi}^{3} \\
\mathbf{q}_{\phi}^{3} \\
\mathbf{q}_{\psi}^{1-4}
\end{array}\right], \mathbf{f}_{g}=\left[\begin{array}{c}
\mathbf{\varphi}_{I I}^{1}{ }^{T} \mathbf{f}_{I}^{1} \\
\mathbf{0} \\
\mathbf{0} \\
\mathbf{0} \\
\boldsymbol{\Psi}_{I B}^{1}{ }^{T} \mathbf{f}_{I}^{1}
\end{array}\right] \\
& \mathbf{M}_{g}=\left[\begin{array}{ccccc}
\mathbf{m}_{\phi \phi}^{1} & \mathbf{0} & \mathbf{0} & \mathbf{0} & \mathbf{m}_{\phi \psi}^{1} \\
\mathbf{0} & \mathbf{m}_{\phi \phi}^{2} & \mathbf{0} & \mathbf{0} & \mathbf{m}_{\phi \psi}^{2} \\
\mathbf{0} & \mathbf{0} & \mathbf{m}_{\phi \phi}^{3} & \mathbf{0} & \mathbf{m}_{\phi \psi}^{3} \\
\mathbf{0} & \mathbf{0} & \mathbf{0} & \mathbf{m}_{\phi \phi}^{4} & \mathbf{m}_{\phi \psi}^{4} \\
\mathbf{m}_{\phi \psi}^{1}{ }^{T} & \mathbf{m}_{\phi \psi}^{2}{ }^{T} & \mathbf{m}_{\phi \psi}^{3}{ }^{3} & \mathbf{m}_{\phi \psi}^{\mathbf{S}^{4}} & \mathbf{m}_{\psi \psi}^{1-4}
\end{array}\right], \mathbf{K}_{g}=\left[\begin{array}{ccccc}
\boldsymbol{\Lambda}_{\phi \phi}^{1} & \mathbf{0} & \mathbf{0} & \mathbf{0} & \mathbf{0} \\
\mathbf{0} & \boldsymbol{\Lambda}_{\phi \phi}^{2} & \mathbf{0} & \mathbf{0} & \mathbf{0} \\
\mathbf{0} & \mathbf{0} & \boldsymbol{\Lambda}_{\phi \phi}^{3} & \mathbf{0} & \mathbf{0} \\
\mathbf{0} & \mathbf{0} & \mathbf{0} & \boldsymbol{\Lambda}_{\phi \phi}^{4} & \mathbf{0} \\
\mathbf{0} & \mathbf{0} & \mathbf{0} & \mathbf{0} & \mathbf{k}_{\psi \psi \psi}^{1-4}
\end{array}\right] .
\end{aligned}
$$

The number of constraint modes in Eq. (9) can be substantially diminished if one solves the generalized eigenproblem associated to its mass and stiffness matrices, (see [25, 26]),

$$
\left[\mathbf{m}_{\psi \psi}^{1-4}-\lambda_{C C}^{i} \mathbf{k}_{\psi \psi}^{1-4}\right] \xi^{i}=0 .
$$

The resulting eigenvectors $\xi^{i}$ are referred to as the Characteristic Constraint (CC) modes, and their number $N_{C C}$ is supposed to be less than the number of constraint modes. The eigenvectors $\xi^{i}$ are normalized to unit generalized mass and can be organized in columns in the matrix $\Xi=\left[\begin{array}{llll}\xi^{1} & \xi^{2} & \ldots & \xi^{N_{c c}}\end{array}\right]$. This matrix can be used to transform from the constraint mode amplitudes $\mathbf{q}_{\psi}^{1-4}$ to the CC ones $\mathbf{q}_{C C}$,

$$
\mathbf{q}_{\psi}^{1-4}=\Xi \mathbf{q}_{C C} .
$$

The stiffness matrix $\mathbf{k}_{\psi \psi}^{1-4}$ diagonalizes to $\boldsymbol{\Lambda}_{C C}=\operatorname{diag}\left[\lambda_{c c}^{i}\right]_{N_{c c} \times N_{c c}}$ and the modal equations (9) can then be rewritten as

$$
\mathbf{M}_{g-C C} \ddot{\mathbf{q}}_{g-C C}+\mathbf{K}_{g-C C} \mathbf{q}_{g-C C}=\mathbf{f}_{g-C C},
$$

with 


$$
\begin{aligned}
& \mathbf{q}_{g-C C}=\left[\begin{array}{c}
\mathbf{q}_{\varphi}^{1} \\
\mathbf{q}_{\varphi}^{2} \\
\mathbf{q}_{\varphi}^{3} \\
\mathbf{q}_{\varphi}^{4} \\
\mathbf{q}_{C C}
\end{array}\right], \mathbf{f}_{g-C C}=\left[\begin{array}{c}
\boldsymbol{\varphi}_{I I}^{1 T} \mathbf{f}_{I}^{1} \\
\mathbf{0} \\
\mathbf{0} \\
\mathbf{0} \\
\Xi^{T} \boldsymbol{\Psi}_{I B}^{1 T} \mathbf{f}_{I}^{1}
\end{array}\right], \mathbf{M}_{g-C C}=\left[\begin{array}{ccccc}
\mathbf{m}_{\varphi \varphi}^{1} & \mathbf{0} & \mathbf{0} & \mathbf{0} & \mathbf{m}_{\varphi \psi}^{1} \Xi \\
\mathbf{0} & \mathbf{m}_{\varphi \varphi}^{2} & \mathbf{0} & \mathbf{0} & \mathbf{m}_{\varphi \psi}^{2} \Xi \\
\mathbf{0} & \mathbf{0} & \mathbf{m}_{\varphi \varphi}^{3} & \mathbf{0} & \mathbf{m}_{\varphi \psi}^{3} \Xi \\
\mathbf{0} & \mathbf{0} & \mathbf{0} & \mathbf{m}_{\varphi \varphi}^{4} & \mathbf{m}_{\varphi \psi}^{4} \Xi \\
\Xi^{T} \mathbf{m}_{\varphi \psi}^{1}{ }^{T} & \Xi^{T} \mathbf{m}_{\varphi \psi}^{2}{ }^{T} & \Xi^{T} \mathbf{m}_{\varphi \psi}^{3{ }^{T}} & \Xi^{T} \mathbf{m}_{\varphi \psi}^{4}{ }^{T} & \mathbf{I}_{c c}^{4}
\end{array}\right], \\
& \mathbf{K}_{g-C C}=\left[\begin{array}{ccccc}
\boldsymbol{\Lambda}_{\varphi \varphi}^{1} & \mathbf{0} & \mathbf{0} & \mathbf{0} & \mathbf{0} \\
\mathbf{0} & \boldsymbol{\Lambda}_{\varphi \varphi}^{2} & \mathbf{0} & \mathbf{0} & \mathbf{0} \\
\mathbf{0} & \mathbf{0} & \boldsymbol{\Lambda}_{\varphi \varphi}^{3} & \mathbf{0} & \mathbf{0} \\
\mathbf{0} & \mathbf{0} & \mathbf{0} & \boldsymbol{\Lambda}_{\varphi \varphi}^{4} & \mathbf{0} \\
\mathbf{0} & \mathbf{0} & \mathbf{0} & \mathbf{0} & \boldsymbol{\Lambda}_{C C}
\end{array}\right]
\end{aligned}
$$

The algebraic matrix system (12) expresses the coupling of the subsystem blocked modes to the $\mathrm{CC}$ ones through mass matrix elements. Expanding the system in rows allows one to recover the standard canonical problem at the basis of some energy-based methods [2],

$$
\begin{gathered}
{\left[\mathbf{m}_{\phi \phi}^{\alpha}\right]_{p p} \ddot{q}_{\phi p}^{\alpha}+\lambda^{p} q_{\phi p}^{\alpha}=\left[\boldsymbol{\varphi}_{I I}^{\alpha^{T}} \mathbf{f}_{I}^{\alpha}\right]_{p} \delta_{1 \alpha}-\sum_{q}\left[\mathbf{m}_{\phi \psi}^{\alpha} \Xi\right]_{p q} \ddot{q}_{C C q}, \quad \alpha=1 \ldots 4,} \\
\ddot{q}_{C C p}+\lambda_{C C}^{i} q_{C C p}=\left\lfloor\Xi^{T} \boldsymbol{\Psi}_{I B}^{{ }^{T}} \mathbf{f}_{I}^{1}\right]_{p}-\sum_{\alpha} \sum_{q}\left\lfloor\Xi^{T} \mathbf{m}_{\phi \psi}^{\alpha}\right]_{p q} \ddot{q}_{\phi q}^{\alpha} .
\end{gathered}
$$

Eq. (13a) states that any normal mode of subsystem $\alpha$, say the $p$-th mode $q_{\phi p}^{\alpha}$, behaves as a mass-spring oscillator (1.h.s of Eq. (13a)) that is mass-coupled to a $q$-th characteristic constraint CC mode (r.h.s of Eq. (13a)) but not to the normal modes of other subsystems. Conversely, Eq. (13b) shows that any CC mode also corresponds to a mass-spring oscillator that exhibits inertial coupling with the normal modes of the four panel subsystems, $\alpha=1 \ldots 4$.

In comparison with the DMF approach to the problem in [7], a fifth subsystem appears in the $\mathrm{BC}$ formulation. It is composed by the $\mathrm{CC}$ modes which are not localized on one panel (see Figure 3, and in particular Figure 3c), but it rather represents the global part of the dynamic behavior of the coupled plates. This subsystem is also directly excited by the external force term $\Xi^{T} \boldsymbol{\psi}_{I B}^{1{ }^{T}} \mathbf{f}_{I}^{1}$ in Eqs. (12) and (13b). 
In addition to being expressed in the canonical form (13), to check if the above equations fulfill with the MCAs it should be verified whether resonant modes could account for the whole energy of the system at a given frequency band. This will be tested for the cases \#1 and \#2 described in section 3. Prior to that, however, we should depict how to compute the subsystem energies from the amplitudes of the normal modes, $\mathbf{q}_{\phi \phi}^{\alpha}$, and CC modes, $\mathbf{q}_{C C}$.

\subsection{Computation of subsystem energies}

The energy of a subsystem is given by the sum of the kinetic and potential energies of the modes in the subsystem. These quantities are averaged in time though this will be not explicitly indicated hereafter.

Assuming a unit harmonic external excitation, $e^{j \omega t}$, and introducing homogeneous structural damping into the system, the modal governing equations (12) transform to

$$
\left[-\omega^{2} \mathbf{M}_{g-C C}+(1+j \eta) \mathbf{K}_{g-C C}\right] \mathbf{q}_{g-C C}(\omega)=\mathbf{f}_{g-C C}(\omega) .
$$

From Eq. (14) and the matrix definitions in Eqs. (11) and (12), the kinetic energy of subsystem $\alpha$ can be computed as,

$$
E_{k}^{\alpha}(\omega)=\frac{1}{4} \omega^{2}\left[\begin{array}{c}
\mathbf{q}_{\phi}^{\alpha} \\
\mathbf{q}_{C C}
\end{array}\right]^{H}\left[\begin{array}{cc}
\mathbf{m}_{\phi \phi}^{\alpha} & \overline{\mathbf{m}}_{\phi \psi}^{\alpha} \\
\overline{\mathbf{m}}_{\phi \psi}^{\alpha^{T}} & \overline{\mathbf{m}}_{\psi \psi}^{\alpha}
\end{array}\right]\left[\begin{array}{c}
\mathbf{q}_{\phi}^{\alpha} \\
\mathbf{q}_{C C}
\end{array}\right]=E_{k, \phi \phi}^{\alpha}(\omega)+E_{k, \psi \psi}^{\alpha}(\omega)+E_{k, \phi \psi}^{\alpha}(\omega),
$$

with $\overline{\mathbf{m}}_{\psi \psi}^{\alpha}=\Xi^{H} \boldsymbol{\psi}_{I B}^{T} \mathbf{m}_{I I}^{\alpha} \boldsymbol{\psi}_{I B} \Xi$ and $\overline{\mathbf{m}}_{\phi \psi}^{\alpha}=\boldsymbol{\psi}_{I B}^{T} \mathbf{m}_{I I}^{\alpha} \boldsymbol{\varphi}_{I I} \Xi$. The three summands in the second equality of (15) are given by,

- $E_{k, \phi \phi}^{\alpha}(\omega)=\frac{1}{4} \omega^{2} \mathbf{q}_{\phi}^{\alpha}{ }^{H} \mathbf{m}_{\phi \phi}^{\alpha} \mathbf{q}_{\phi}^{\alpha}:$ kinetic energy from the normal modes,

- $E_{k, \psi \psi}^{\alpha}(\omega)=\frac{1}{4} \omega^{2} \mathbf{q}_{C C}^{H} \overline{\mathbf{m}}_{\psi \psi}^{\alpha} \mathbf{q}_{C C}$ : kinetic energy associated to the CC modes,

- $E_{k, \phi \psi}^{\alpha}(\omega)=\frac{1}{2} \omega^{2} \operatorname{Re}\left\{\mathbf{q}_{\phi}^{\alpha^{H}} \overline{\mathbf{m}}_{\phi \psi}^{\alpha} \mathbf{q}_{C C}\right\}:$ kinetic energy arising from the cross interaction between the normal and CC modes.

Notice that $E_{k, \phi \psi}^{\alpha}(\omega)$ can be either positive or negative. This term is a consequence of the fact that normal and $\mathrm{CC}$ modes are not orthogonal with respect to the subsystem mass matrix. 
On the other hand, the time-averaged potential energies of a subsystem can be obtained from

$$
E_{p}^{\alpha}(\omega)=\frac{1}{4}\left[\begin{array}{c}
\mathbf{q}_{\phi}^{\alpha} \\
\mathbf{q}_{C C}
\end{array}\right]^{H}\left[\begin{array}{cc}
\boldsymbol{\Lambda}_{\phi \phi}^{\alpha} & \mathbf{0} \\
\mathbf{0} & \overline{\mathbf{k}}_{\psi \psi}^{\alpha}
\end{array}\right]\left[\begin{array}{c}
\mathbf{q}_{\phi}^{\alpha} \\
\mathbf{q}_{C C}
\end{array}\right]=E_{p, \phi \phi}^{\alpha}(\omega)+E_{p, \psi \psi}^{\alpha}(\omega),
$$

with $\overline{\mathbf{k}}_{\psi \psi}^{\alpha}=\Xi^{H} \boldsymbol{\psi}_{I B}^{T} \mathbf{k}_{I I}^{\alpha} \boldsymbol{\psi}_{I B} \Xi$. The two summands in the r.h.s of Eq. (16) correspond to,

- $E_{p, \phi \phi}^{\alpha}(\omega)=\frac{1}{4} \mathbf{q}_{\phi}^{\alpha^{H}} \boldsymbol{\Lambda}_{\phi \phi}^{\alpha} \mathbf{q}_{\phi}^{\alpha}$ : potential energy from the normal modes,

- $E_{p, \psi \psi}^{\alpha}(\omega)=\frac{1}{4} \mathbf{q}_{C C}^{H} \overline{\mathbf{k}}_{\psi \psi}^{\alpha} \mathbf{q}_{C C}$ : potential energy associated to the CC modes.

The mean energy of a subsystem in response to a white noise force of unit power spectral density in each octave band is obtained by integrating the summation of the kinetic (15) and potential (16) spectral energies over the bandwidth using a simple quadrature rule.

\subsection{CB numerical simulations and discussion}

The total energies of the subsystems have been computed with Eq. (15-16) for test cases $\# 1$ and \#2. The energies have been integrated in the $1 \mathrm{kHz}$ octave band and compared to a reference solution obtained from the global mode computation described in section 2.2 of [28]. 200 normal modes have been considered for the modal energy computations, which has proved more than enough to ensure convergence. As regards the $\mathrm{CC}$ modes, 20 of them have sufficed to achieve a good agreement with the reference solution. This is remarkable since 396 constraint modes would have been necessary to achieve similar results.

Results are given in Table 1 for the two test cases. As observed, the total energies estimated with the $\mathrm{CB}$ approach are compliant with the reference ones. Except for the excited subsystem, where the contribution of the normal modes dominates, the normal modes and the $\mathrm{CC}$ ones equally add to the energies of panels 2, 3 and 4 . There are, however, some issues that prevent one identifying Eq. (11) as a suitable candidate to fulfill the MCAs. On the one hand the resonant modes contribute significantly to the overall energy of the normal modes, especially for subsystem 1, but hardly exceed 50-60\% of the energy for subsystems 2, 3 and 4 (bear in mind that results are presented in $\mathrm{dB}$ in Table 1). On the other hand, the energy cross contributions are rather important (mainly for test case \#1) and attain negative values. An energy-based model like SEA or SmEdA cannot account for 
these cross contributions which arise because the subsystem normal modes and the CCmodes are not orthogonal with respect to the subsystem mass matrix. Indeed, and as seen from the MCAs in section 2.1, in those methods modes are identified with oscillators and the power flow interchanged between them is proportional to the difference between their energies. The energy of each mode can be estimated, but not the cross-contribution between them. Moreover, in the results of Table 1 the cross contributions also include the influence of non-resonant modes.

To further check the degree of compliance with the MCAs, we have made a second computation retaining only the resonant normal modes (and also the $20 \mathrm{CC}$-modes). The amount of resonant normal modes for each plate is given in Table 2. As observed, the number is much lower than that of the previous computation, where 200 normal modes per plate were considered. Yet, the number still complies with the SEA requirements of at least 10 subsystem modes for frequency band. Table 2 also includes the modal overlap factors deduced from the number of resonant modes. The modal overlap values are close to one, which indicates that the dynamic behavior of the subsystems is neither non-diffuse (as for high modal overlaps), nor dominated by a few strongly resonant modes (as for small modal overlaps). Therefore, the conditions for SEA application are fulfilled [2].

The results with the sole consideration of resonant modes are shown in Table 3. The energy predictions are clearly less accurate than those from Table 1. Actually, only the energy of the excited panel is correctly estimated. For panels 2, 3 and 4, this second CB calculation underestimates the energy levels between $1 \mathrm{~dB}$ and $4.5 \mathrm{~dB}$, depending on the subsystem and the test case. The influence of the cross terms has also noticeably decreased because of the exclusion of the non-resonant normal modes.

To better understand the reason of the discrepancies between the second and first calculations, an analysis of the modal parameters (modal injected power, modal coupling mass, modal energy) of the first CB computation has been performed. If we arbitrarily focus on subsystem 3 (equivalent results arise for the other non-excited subsystems), some hints can be grasped on what is happening. One can observe in Figure 4a that the low frequency non-resonant modes outside the $1 \mathrm{kHz}$ band have substantial energy. In particular, the first mode (which is clearly non-resonant) has the largest energy of all modes. This is in contrast with the global distribution of the modal energy one gets form 
the DMF approach (compare with Figure 4b in [7]). In that figure, the most energetic modes are mainly resonant which allows one neglecting them in the dual modal formulation without introducing much error.

We can gather more information having a look at the modal mass coupling matrix $\mathbf{m}_{\phi \psi}^{3} \Xi$ in Figure $4 \mathrm{~b}$ of the current paper. The first non-resonant normal mode appears strongly coupled with the first CC-mode. Similarly, other non-resonant normal modes exhibit substantial couplings with the $\mathrm{CC}$ ones. This is at the origin the problem. The strong coupling makes the non-resonant modes play a significant role in the energy sharing between subsystems. Consequently, they cannot be neglected, and the MCAs may be violated if the standard CB formulation Eq. (11) is considered. To somewhat overcome this problem, a reformulation of the $\mathrm{CB}$ equations will be next proposed.

\begin{tabular}{|c|c|c|c|c|c|}
\hline $\begin{array}{l}\text { Test } \\
\text { case }\end{array}$ & Energy $\quad$ Panel & 1 & 2 & 3 & 4 \\
\hline$\# 1$ & Reference & $68.5 \mathrm{~dB}$ & $61.4 \mathrm{~dB}$ & $44.7 \mathrm{~dB}$ & $46.4 \mathrm{~dB}$ \\
\hline$\# 1$ & $\begin{aligned} \text { CB - Normal modes } \\
\text { (Resonant contrib.) }\end{aligned}$ & $\begin{array}{l}68.1 \mathrm{~dB} \\
(67.5 \mathrm{~dB})\end{array}$ & $\begin{array}{l}60.5 \mathrm{~dB} \\
(57.7 \mathrm{~dB})\end{array}$ & $\begin{array}{l}47.1 \mathrm{~dB} \\
(44.2 \mathrm{~dB})\end{array}$ & $\begin{array}{l}47.7 \mathrm{~dB} \\
(46.1 \mathrm{~dB})\end{array}$ \\
\hline$\# 1$ & $\mathrm{CB}-\mathrm{CC}$ modes & $60.8 \mathrm{~dB}$ & $60.5 \mathrm{~dB}$ & $44.2 \mathrm{~dB}$ & $42.8 \mathrm{~dB}$ \\
\hline$\# 1$ & CB - Cross contributions & $57.8 \mathrm{~dB}(-)$ & $59.2 \mathrm{~dB}(-)$ & $46.8 \mathrm{~dB}(-)$ & $45.3 \mathrm{~dB}(-)$ \\
\hline$\# 1$ & CB - Total & $68.5 \mathrm{~dB}$ & $61.5 \mathrm{~dB}$ & $44.7 \mathrm{~dB}$ & $46.4 \mathrm{~dB}$ \\
\hline$\# 2$ & Reference & $67.9 \mathrm{~dB}$ & $57.2 \mathrm{~dB}$ & $56.6 \mathrm{~dB}$ & $57.2 \mathrm{~dB}$ \\
\hline$\# 2$ & $\begin{aligned} \text { CB }- \text { Normal modes } \\
\text { (Resonant contrib.) }\end{aligned}$ & $\begin{array}{l}67.8 \mathrm{~dB} \\
(67.4 \mathrm{~dB})\end{array}$ & $\begin{array}{l}56.1 \mathrm{~dB} \\
(53.8 \mathrm{~dB})\end{array}$ & $\begin{array}{l}54.9 \mathrm{~dB} \\
(52.8 \mathrm{~dB})\end{array}$ & $\begin{array}{l}54.9 \mathrm{~dB} \\
(53.5 \mathrm{~dB})\end{array}$ \\
\hline$\# 2$ & $\mathrm{CB}-\mathrm{CC}$ modes & $55.9 \mathrm{~dB}$ & $55.6 \mathrm{~dB}$ & $55.2 \mathrm{~dB}$ & $54.9 \mathrm{~dB}$ \\
\hline$\# 2$ & CB - Cross contributions & $50.0 \mathrm{~dB}(-)$ & $54.0 \mathrm{~dB}(-)$ & $52.9 \mathrm{~dB}(-)$ & $49.8 \mathrm{~dB}(-)$ \\
\hline$\# 2$ & CB - Total & $67.9 \mathrm{~dB}$ & $57.2 \mathrm{~dB}$ & $56.6 \mathrm{~dB}$ & $57.2 \mathrm{~dB}$ \\
\hline
\end{tabular}

Table 1: Subsystem energies and different contributions resulting from the standard CB method $\left(\mathrm{dB}\right.$, ref. $10^{-12} \mathrm{~J},(-)=$ negative contributions). Results for the octave band $1 \mathrm{kHz}$.

\begin{tabular}{|c|l|l|l|l|l|}
\hline $\begin{array}{c}\text { Test } \\
\text { case }\end{array}$ & Quantity & \multicolumn{1}{|c|}{1} & \multicolumn{1}{|c|}{2} & \multicolumn{1}{|c|}{3} & \multicolumn{1}{|c|}{} \\
\hline$\# 1$ & Number of resonant modes & 38 & 27 & 101 & 78 \\
\hline$\# 1$ & Modal overlap factor & 1.1 & 0.8 & 2.8 & 2.2 \\
\hline$\# 2$ & Number of resonant modes & 38 & 27 & 24 & 17 \\
\hline$\# 2$ & Modal overlap factor & 1.1 & 0.8 & 0.7 & 0.5 \\
\hline
\end{tabular}

Table 2: Number of resonant modes and modal overlap factor for each panel. 


\begin{tabular}{|c|c|c|c|c|c|}
\hline $\begin{array}{l}\text { Test } \\
\text { case }\end{array}$ & Energy $\quad$ Panel & 1 & 2 & 3 & 4 \\
\hline$\# 1$ & Reference & $68.5 \mathrm{~dB}$ & $61.4 \mathrm{~dB}$ & $44.7 \mathrm{~dB}$ & $46.4 \mathrm{~dB}$ \\
\hline$\# 1$ & $\mathrm{CB}-\mathrm{R}$ normal modes & $67.6 \mathrm{~dB}$ & $56.2 \mathrm{~dB}$ & $38.8 \mathrm{~dB}$ & $43.0 \mathrm{~dB}$ \\
\hline$\# 1$ & $\mathrm{CB}-\mathrm{CC}$ modes & $54.7 \mathrm{~dB}$ & $54.7 \mathrm{~dB}$ & $34.2 \mathrm{~dB}$ & $33.9 \mathrm{~dB}$ \\
\hline$\# 1$ & $\mathrm{CB}-$ Cross contributions & $36.9 \mathrm{~dB}(-)$ & $39.9 \mathrm{~dB}(-)$ & $21.9 \mathrm{~dB}(-)$ & $26.3 \mathrm{~dB}(-)$ \\
\hline$\# 1$ & CB - Total & $67.8 \mathrm{~dB}$ & $58.5 \mathrm{~dB}$ & $40.1 \mathrm{~dB}$ & $43.4 \mathrm{~dB}$ \\
\hline$\# 2$ & Reference & $67.9 \mathrm{~dB}$ & $57.2 \mathrm{~dB}$ & $56.6 \mathrm{~dB}$ & $57.2 \mathrm{~dB}$ \\
\hline$\# 2$ & $\mathrm{CB}-\mathrm{R}$ normal modes & $67.5 \mathrm{~dB}$ & $54.7 \mathrm{~dB}$ & $49.5 \mathrm{~dB}$ & $52.3 \mathrm{~dB}$ \\
\hline \#2 & $\mathrm{CB}-\mathrm{CC}$ modes & $51.8 \mathrm{~dB}$ & $51.8 \mathrm{~dB}$ & $51.8 \mathrm{~dB}$ & $51.7 \mathrm{~dB}$ \\
\hline$\# 2$ & $\mathrm{CB}$ - Cross contributions & $39.1 \mathrm{~dB}(-)$ & $37.7 \mathrm{~dB}(-)$ & $36.4 \mathrm{~dB}(-)$ & $38.8 \mathrm{~dB}(-)$ \\
\hline$\# 2$ & CB - Total & $67.6 \mathrm{~dB}$ & $56.5 \mathrm{~dB}$ & $53.7 \mathrm{~dB}$ & $55.1 \mathrm{~dB}$ \\
\hline
\end{tabular}

Table 3: Same type of results than in Table 1 with the standard CB method but only considering the Resonant (R) normal modes. Results for the octave band $1 \mathrm{kHz}$.

\section{Reformulation of the Craig-Bampton method}

\subsection{Reformulated CB modal equations for four subsystems coupled at a junction}

Let us start by splitting the modal amplitude vector $\mathbf{q}_{g}$ in Eq. (9) in two parts: $\mathbf{q}_{g}^{R}$ which includes the amplitude vectors of the resonant (R) normal modes, and $\mathbf{q}_{g}^{N R+I}$ standing for the amplitude vectors of the non-resonant (NR) normal modes, plus the constraint interface (I) modes (NR+I modes). The size of this last vector corresponds to the summation of the number of NR modes, $N_{N R}$, and the number of the constraint modes (i.e. the number of boundary d.o.f.s). The algebraic system of equations (9) can then be rewritten as,

$$
\left[\begin{array}{cc}
\mathbf{M}_{g}^{R} & \mathbf{M}_{g}^{R / N R+I} \\
\mathbf{M}_{g}^{R / N R+I} & \mathbf{M}_{g}^{N R+I}
\end{array}\right]\left[\begin{array}{c}
\ddot{\mathbf{q}}_{g}^{R} \\
\ddot{\mathbf{q}}_{g}^{N R+I}
\end{array}\right]+\left[\begin{array}{cc}
\boldsymbol{\Lambda}_{g}^{R} & \mathbf{0} \\
\mathbf{0} & \mathbf{K}_{g}^{N R+I}
\end{array}\right]\left[\begin{array}{c}
\mathbf{q}_{g}^{R} \\
\mathbf{q}_{g}^{N R+I}
\end{array}\right]=\left[\begin{array}{c}
\mathbf{f}_{g}^{R} \\
\mathbf{f}_{g}^{N R+I}
\end{array}\right],
$$

with 


$$
\begin{aligned}
& \mathbf{M}_{g}^{R}=\left[\begin{array}{cccc}
\mathbf{m}_{\phi \phi}^{1-R} & \mathbf{0} & \mathbf{0} & \mathbf{0} \\
\mathbf{0} & \mathbf{m}_{\phi \phi}^{2-R} & \mathbf{0} & \mathbf{0} \\
\mathbf{0} & \mathbf{0} & \mathbf{m}_{\phi \phi}^{3-R} & \mathbf{0} \\
\mathbf{0} & \mathbf{0} & \mathbf{0} & \mathbf{m}_{\phi \phi}^{4-R}
\end{array}\right], \mathbf{M}_{g}^{N R+I}=\left[\begin{array}{ccccc}
\mathbf{m}_{\phi \phi}^{1-N R} & \mathbf{0} & \mathbf{0} & \mathbf{0} & \mathbf{m}_{\phi \psi}^{1-N R} \\
\mathbf{0} & \mathbf{m}_{\phi \phi}^{2-N R} & \mathbf{0} & \mathbf{0} & \mathbf{m}_{\phi \psi}^{2-N R} \\
\mathbf{0} & \mathbf{0} & \mathbf{m}_{\phi \phi}^{3-N R} & \mathbf{0} & \mathbf{m}_{\phi \psi}^{3-N R} \\
\mathbf{0} & \mathbf{0} & \mathbf{0} & \mathbf{m}_{\phi \phi}^{4-N R} & \mathbf{m}_{\phi \psi}^{4-N R} \\
\mathbf{m}_{\phi \psi}^{1-N R^{T}} & \mathbf{m}_{\phi \psi}^{2-N R^{T}} & \mathbf{m}_{\phi \psi}^{3-N R^{T}} & \mathbf{m}_{\phi \psi}^{4-N R^{T}} & \mathbf{m}_{\psi \psi}^{1-4}
\end{array}\right], \\
& \boldsymbol{\Lambda}_{g}^{R}=\left[\begin{array}{cccc}
\boldsymbol{\Lambda}_{\phi \phi}^{1-R} & \mathbf{0} & \mathbf{0} & \mathbf{0} \\
\mathbf{0} & \boldsymbol{\Lambda}_{\phi \phi}^{2-R} & \mathbf{0} & \mathbf{0} \\
\mathbf{0} & \mathbf{0} & \boldsymbol{\Lambda}_{\phi \phi}^{3-R} & \mathbf{0} \\
\mathbf{0} & \mathbf{0} & \mathbf{0} & \boldsymbol{\Lambda}_{\phi \phi}^{4-R}
\end{array}\right], \mathbf{K}_{g}^{N R+I}=\left[\begin{array}{ccccc}
\boldsymbol{\Lambda}_{\phi \phi}^{1-N R} & \mathbf{0} & \mathbf{0} & \mathbf{0} & \mathbf{0} \\
\mathbf{0} & \boldsymbol{\Lambda}_{\phi \phi}^{2-N R} & \mathbf{0} & \mathbf{0} & \mathbf{0} \\
\mathbf{0} & \mathbf{0} & \boldsymbol{\Lambda}_{\phi \phi}^{3-N R} & \mathbf{0} & \mathbf{0} \\
\mathbf{0} & \mathbf{0} & \mathbf{0} & \boldsymbol{\Lambda}_{\phi \phi}^{4-N R} & \mathbf{0} \\
\mathbf{0} & \mathbf{0} & \mathbf{0} & \mathbf{0} & \mathbf{k}_{\psi \psi}^{1-4}
\end{array}\right], \\
& \mathbf{M}_{g}^{R / N R+I}=\left[\begin{array}{ccccc}
\mathbf{0} & \mathbf{0} & \mathbf{0} & \mathbf{0} & \mathbf{m}_{\phi \psi}^{1-R} \\
\mathbf{0} & \mathbf{0} & \mathbf{0} & \mathbf{0} & \mathbf{m}_{\phi \psi}^{2-R} \\
\mathbf{0} & \mathbf{0} & \mathbf{0} & \mathbf{0} & \mathbf{m}_{\phi \psi}^{3-R} \\
\mathbf{0} & \mathbf{0} & \mathbf{0} & \mathbf{0} & \mathbf{m}_{\phi \psi}^{4-R}
\end{array}\right], \mathbf{q}_{g}^{N R+I}=\left[\begin{array}{c}
\mathbf{q}_{\phi}^{1-N R} \\
\mathbf{q}_{\phi}^{2-N R} \\
\mathbf{q}_{\phi}^{3-N R} \\
\mathbf{q}_{\phi}^{4-N R} \\
\mathbf{q}_{\psi}^{1-4}
\end{array}\right], \mathbf{q}_{g}^{R}=\left[\begin{array}{c}
\mathbf{q}_{\phi}^{1-R} \\
\mathbf{q}_{\phi}^{2-R} \\
\mathbf{q}_{\phi}^{3-R} \\
\mathbf{q}_{\phi}^{4-R}
\end{array}\right], \mathbf{f}_{g}^{N R+I}=\left[\begin{array}{c}
\boldsymbol{\varphi}_{I I}^{1-N R^{T}} \mathbf{f}_{I}^{1} \\
\mathbf{0} \\
\mathbf{0} \\
\mathbf{0} \\
\boldsymbol{\psi}_{I B}^{1}{ }^{T} \mathbf{f}_{I}^{1}
\end{array}\right], \mathbf{f}_{g}^{R}=\left[\begin{array}{c}
\boldsymbol{\varphi}_{I I}^{1-R^{T}} \mathbf{f}_{I}^{1} \\
\mathbf{0} \\
\mathbf{0} \\
\mathbf{0}
\end{array}\right]
\end{aligned}
$$

Next, and similarly to what was done for the calculation of the $\mathrm{CC}$ modes in Eq. (10), an eigen-analysis is carried out for the matrices related to the NR+I modes,

$$
\left[\mathbf{K}_{g}^{N R+I}-\lambda_{C}^{i} \mathbf{M}_{g}^{N R+I}\right] \zeta^{i}=0 .
$$

The eigenvectors $\zeta^{i}$ associated to the eigenvalues $\lambda_{C}^{i}$ will be hereafter referred to as the Coupling (C) modes. It should be remarked that the definition of the NR modes depends on the considered frequency band. Consequently, the $\mathrm{C}$ modes will also depend on it.

Suppose a total of $N_{C}$ coupling modes. Their eigenvectors can be settled in columns in a matrix $\Gamma=\left[\zeta^{1} \zeta^{2} \ldots \zeta^{N_{C}}\right]$. The modal coordinate vector $\mathbf{q}_{g}$ can then be projected onto the $\mathrm{C}$ modes basis through the transformation

$$
\mathbf{q}_{g}=\left[\begin{array}{c}
\mathbf{q}_{g}^{R} \\
\mathbf{q}_{g}^{N R+I}
\end{array}\right]=\mathbf{C}\left[\begin{array}{c}
\mathbf{q}_{g}^{R} \\
\mathbf{q}_{g}^{C}
\end{array}\right] \text {, with } \mathbf{C}=\left[\begin{array}{cc}
\mathbf{I} & \mathbf{0} \\
\mathbf{0} & \boldsymbol{\Gamma}
\end{array}\right] .
$$

Pre-and post-multiplication of Eq. (17) by $\mathbf{C}$ results in

$$
\left[\begin{array}{cc}
\mathbf{M}_{g}^{R} & \mathbf{M}_{g}^{R / C} \\
\mathbf{M}_{g}^{R / C^{T}} & \mathbf{M}_{g}^{C}
\end{array}\right]\left[\begin{array}{c}
\ddot{\mathbf{q}}_{g}^{R} \\
\ddot{\mathbf{q}}_{g}^{C}
\end{array}\right]+\left[\begin{array}{cc}
\boldsymbol{\Lambda}_{g}^{R} & \mathbf{0} \\
\mathbf{0} & \boldsymbol{\Lambda}^{C}
\end{array}\right]\left[\begin{array}{c}
\mathbf{q}_{g}^{R} \\
\mathbf{q}_{g}^{C}
\end{array}\right]=\left[\begin{array}{c}
\mathbf{f}_{g}^{R} \\
\mathbf{f}_{g}^{C}
\end{array}\right]
$$


with $\mathbf{M}_{g}^{C}=\Gamma^{T} \mathbf{M}_{g}^{N R+I} \boldsymbol{\Gamma}, \mathbf{M}_{g}^{R / C}=\mathbf{M}_{g}^{R / N R+I} \boldsymbol{\Gamma}$ and $\mathbf{f}_{g}^{C}=\boldsymbol{\Gamma}^{T} \mathbf{f}_{g}^{N R+I}$. As in Eq. (13), we could expand Eq. (20) and check that this system essentially establishes an inertial coupling between the resonant subsystem modes and the $\mathrm{C}$ modes. The subsystem energies can be calculated following the procedure exposed in section 4.2 .

\subsection{Reformulated CB numerical simulations and discussion}

The reformulated CB equations have been applied once more to test cases \#1 and \#2. 815 and $951 \mathrm{C}$ modes below $20 \mathrm{kHz}$ have been respectively used for them. The resulting modal contributions to the subsystem energies are summarized in Table 4 and show very good agreement with the reference solution. For instance, the energy of panel P3 is correctly predicted for tests \#1 and \#2. This was not the case when only R normal modes were considered in the standard $\mathrm{CB}$ equations (see Table 3). Also, the cross contributions turn to be negligible compared with the contributions of the $\mathrm{R}$ normal modes and the $\mathrm{C}$ modes. Hence, the latter suffice to well approximate the subsystem energies.

\begin{tabular}{|c|c|c|c|c|c|}
\hline $\begin{array}{l}\text { Test } \\
\text { case }\end{array}$ & Energy $\quad$ Panel & 1 & 2 & 3 & 4 \\
\hline$\# 1$ & Reference & $68.5 \mathrm{~dB}$ & $61.4 \mathrm{~dB}$ & $44.7 \mathrm{~dB}$ & $46.4 \mathrm{~dB}$ \\
\hline$\# 1$ & CB - R Normal modes & $67.5 \mathrm{~dB}$ & $57.7 \mathrm{~dB}$ & $44.2 \mathrm{~dB}$ & $46.1 \mathrm{~dB}$ \\
\hline$\# 1$ & $\mathrm{CB}-\mathrm{C}$ modes & $59.7 \mathrm{~dB}$ & $59.2 \mathrm{~dB}$ & $34.8 \mathrm{~dB}$ & $34.8 \mathrm{~dB}$ \\
\hline$\# 1$ & CB - Cross contributions & $51.4 \mathrm{~dB}$ & $46.1 \mathrm{~dB}(-)$ & $27.3 \mathrm{~dB}(-)$ & $29.3 \mathrm{~dB}$ \\
\hline$\# 1$ & CB - Total & $68.3 \mathrm{~dB}$ & $61.4 \mathrm{~dB}$ & $44.7 \mathrm{~dB}$ & $46.4 \mathrm{~dB}$ \\
\hline$\# 2$ & Reference & $67.9 \mathrm{~dB}$ & $57.2 \mathrm{~dB}$ & $56.6 \mathrm{~dB}$ & $57.2 \mathrm{~dB}$ \\
\hline$\# 2$ & $\mathrm{CB}-\mathrm{R}$ Normal modes & $67.4 \mathrm{~dB}$ & $54.1 \mathrm{~dB}$ & $52.8 \mathrm{~dB}$ & $53.5 \mathrm{~dB}$ \\
\hline$\# 2$ & $\mathrm{CB}-\mathrm{C}$ modes & $56.0 \mathrm{~dB}$ & $54.3 \mathrm{~dB}$ & $54.2 \mathrm{~dB}$ & $54.2 \mathrm{~dB}$ \\
\hline$\# 2$ & CB - Cross contributions & $49.1 \mathrm{~dB}$ & $39.9 \mathrm{~dB}(-)$ & $39.8 \mathrm{~dB}(-)$ & $40.6 \mathrm{~dB}$ \\
\hline$\# 2$ & CB - Total & $67.8 \mathrm{~dB}$ & $57.1 \mathrm{~dB}$ & $56.5 \mathrm{~dB}$ & $57.0 \mathrm{~dB}$ \\
\hline
\end{tabular}

Table 4: Same type of results than in Table 1 but with the reformulated CB calculation.

The results in Table 4 are mainly consistent with the MCAs except for one question. The $\mathrm{C}$ modes contemplated in the computations contain both, resonant and non-resonant modes. Thus, a new calculation for test case \#2 has been carried out only taking into account the six existing resonant $\mathrm{C}$ modes. Table 5 (second row) indicates that subsystem energies are worse estimated in this occasion. Discrepancies between 1 and $2 \mathrm{~dB}$ can be appreciated (compare with first row in Table 5). 
To better comprehend these outcomes, the modal energy distributions of subsystems 2 and 3, obtained with the sole consideration of resonant modes, are compared in Figure 5 with those from the original CB formulation, which also include non-resonant modes (herein considered as the reference solution). As seen in the figure, the energies of most modes are correctly predicted with the reformulated CB model, even if only resonant $\mathrm{C}$ modes are involved. However, some large discrepancies can also be observed for a few other modes, their energy levels being generally underestimated. For instance, the calculation for the 34-th mode of subsystem 2 provides an energy of $5.0 \times 10^{-10} \mathrm{~J}$, whereas the reference value is $1.9 \times 10^{-8} \mathrm{~J}$. These energy differences in some resonant modes are at the origin of the slight discrepancies in $\mathrm{dB}$ of the subsystem energies.

\begin{tabular}{|l|l|l|l|l|}
\hline Reference & 67.9 dB & $\mathbf{5 7 . 2 ~ d B}$ & $\mathbf{5 6 . 6} \mathbf{d B}$ & $\mathbf{5 7 . 2} \mathbf{d B}$ \\
\hline CB with all the C modes & $67.8 \mathrm{~dB}$ & $57.1 \mathrm{~dB}$ & $56.5 \mathrm{~dB}$ & $57.0 \mathrm{~dB}$ \\
\hline CB with only resonant C modes & $67.8 \mathrm{~dB}$ & $55.8 \mathrm{~dB}$ & $55.3 \mathrm{~dB}$ & $55.7 \mathrm{~dB}$ \\
\hline CB with the selected C modes & $67.7 \mathrm{~dB}$ & $57.1 \mathrm{~dB}$ & $56.4 \mathrm{~dB}$ & $56.8 \mathrm{~dB}$ \\
\hline
\end{tabular}

Table 5. Subsystem energy results for the reformulated CB calculation with various sets of $\mathrm{C}$ modes. Test case \#2.

An analysis of the mass coupling matrix between the R normal modes and the $\mathrm{C}$ modes reveals that some non-resonant $\mathrm{C}$ modes are strongly coupled to the former. A final computation is thus presented which, along with the resonant $\mathrm{C}$ modes, also includes those non-resonant $\mathrm{C}$ modes strongly coupled with the resonant normal modes. A non-resonant $\mathrm{C}$ mode and a resonant normal mode are assumed to be strongly coupled if their coupling mass coefficient in $\mathbf{M}_{g}^{R / C}$ is greater than 0.1 (remember that the normal modes and the $\mathrm{C}$ modes are normalized to unit modal mass). Following this criterion, a total of $198 \mathrm{C}$ modes (6 resonant plus 192 non-resonant) are included in the computation. This supposes neglecting a large quantity of non-resonant C-modes (753 out of 951). The subsystem energies resulting from the reformulated $\mathrm{CB}$ with these selected non-resonant modes are presented in Table 5 (third row), while the corresponding modal energy distributions are shown in Figure 6. A very good agreement with the reference solution is obtained.

To conclude the analysis based on the reformulated CB method, a schematic representation of the proposed modal coupling final arrangement is presented in Figure 7. 
In the diagram, all modes (subsystem normal modes and $\mathrm{C}$ modes) can be associated to oscillators, as demanded by the MCAs. The external force directly excites the resonant normal modes of subsystem 1 and the $\mathrm{C}$ modes. The former are coupled to the latter through mass elements, which fulfil the conservative coupling condition of the MCAs. However, for an accurate prediction of the energy sharing between subsystems it becomes necessary to include some non-resonant $\mathrm{C}$ modes in the model. This point cannot be accounted by SEA and SmEdA, though it is not exclusive of the situation analyzed in this paper. For instance, non-resonant transmission is needed to model energy transfer between acoustic cavities separated by a panel, below the critical frequency (see e.g., [29-30]). That issue is however out of the scope of the current work.

\section{Conclusions}

In this paper we have explored the potential of the Craig-Bampton (CB) method to characterize the coupling between multiple subsystems at a junction, in accordance with the coupling modal assumptions (MCAs) of some energy-based methods. This paper constitutes a continuation of the previous work by the authors in [7], where the dual modal formulation (DMF) was investigated to tackle the same problem. Although the DMF performs well when there is a strong impedance mismatch between subsystems at the junction, it fails when the involved subsystems have similar stiffness. This motivated resorting to the $\mathrm{CB}$ to address that situation.

The CB method is appealing at first sight because it considers normal modes with fixed-interfaces, which could be well adapted for subsystems with comparable stiffness (in this case every subsystem is blocked at the junction by the others). However, despite the developments based on the $\mathrm{CB}$ have been shown to fulfil various of the requisites in the MCAs, numerical tests have revealed that the cross contributions between the subsystem normal modes and the characteristic constraint (CC) modes are not negligible, and that non-resonant low frequency modes must be taken into account to achieve accurate energy predictions. To overcome those difficulties, a reformulation of the $\mathrm{CB}$ modal equations has been proposed.

The reformulated CB method has led to the definition of the coupling $(\mathrm{C})$ modes for a given frequency band of excitation. $\mathrm{C}$ modes are obtained solving a generalized eigenvalue problem composed by the mass and stiffness matrices of the non-resonant 
normal modes and the constraint modes. The cross contributions have been shown to become negligible with the reformulation, thus improving the performance of the original CB method. Notwithstanding the enhancement, the influence of those non-resonant $\mathrm{C}$ modes strongly coupled to the normal subsystem modes cannot be neglected to get precise subsystem energies. The standard SEA or SmEdA methods cannot embed such behavior, but the situation is not so strange, and one can encounter it for instance, in panel sound radiation below the critical frequency. Developments to include non-resonant transmission have been developed in those theories and could be adapted as well to deal with the problem in this paper. Future work may also involve handling the effects of longitudinal and shear waves [31-33], which have been disregarded in this paper, as well as extracting coupling loss factors from the herein reported modal coupling scheme.

\section{REFERENCES}

[1] R.H. Lyon, R.G. Dejong, Theory and application of statistical energy analysis, Second ed. - Boston: Butterworth-Heinemann, 1995. 277p.

[2] A. Le Bot, Foundation of statistical energy analysis in vibroacoustics, First ed. Oxford University Press, Oxford, UK, 2015.

[3] S.H. Crandall, R. Lotz, On the coupling loss factor in statistical energy analysis. The Journal of the Acoustical Society of America, 49 (1) (1971) 352-356.

[4] M.J. Crocker, A.J. Price, Sound transmission using Statistical Energy Analysis, Journal of Sound and Vibration, 9 (1969), 469-486.

[5] R.S. Langley, P.J. Shorter, The wave transmission coefficients and coupling loss factors of point connected structures, Journal of the Acoustical Society of America 113 (2003) 1947-1964.

[6] C. Díaz-Cereceda, J. Poblet-Puig, A. Rodríguez-Ferran, Numerical estimation of coupling loss factors in building acoustics, Journal of Sound and Vibration, 332 (2013) $5433-5450$.

[7] L. Maxit, O. Guasch, A dual modal formulation for multiple flexural subsystems connected at a junction in energy-based models, Mechanical Systems and Signal Processing, Submitted (2018). 
[8] F. Fahy, Vibration of containing structures by sound in the contained fluid. Journal of Sound and Vibration, 10 (1969) 490-512.

[9] F. Fahy, Response of a cylinder to random sound in the contained fluid, Journal of Sound and Vibration, 13 (1970) 171-194.

[10] D. Karnopp, Coupled vibratory-system analysis, using the dual formulation. The Journal of the Acoustical Society of America, 40 (1966) 380-384.

[11] L. Maxit, J.-L. Guyader, Estimation of SEA coupling loss factors using a dual formulation and FEM modal information. Part I: Theory, Journal of Sound and Vibration, 5 (2001) 907-930.

[12] L. Maxit, Reformulation and extension of SEA by taking modal energy distribution into account, PhD thesis, INSA Lyon, 2000 (in French).

[13] A. Le Bot, Derivation of statistical energy analysis from radiative exchanges, Journal of Sound and Vibration 300 (3) (2007) 763-779.

[14] G. Tanner, Dynamical energy analysis-Determining wave energy distributions in vibro-acoustical structures in the high-frequency regime, Journal of Sound and Vibration 320 (4) (2009) 1023-1038.

[15] B. Mace, Statistical energy analysis, energy distribution models and system modes, Journal of Sound and Vibration, 264 (2003) 391-409.

[16] J.L. Guyader, C. Boisson, C. Lesueur, Energy transmission in finite coupled plates, Part I: theory, Journal of Sound and Vibration 81 (1982) 81-92.

[17] S. De Rosa, F. Franco, A scaling procedure for the response of an isolated system with high modal overlap factor, Mechanical Systems and Signal Processing, 22 (2008) 15491565.

[18] S. De Rosa, F. Franco, On the use of the asymptotic scaled modal analysis for timeharmonic structural analysis and for the prediction of coupling loss factors for similar systems, Mechanical Systems and Signal Processing, 24 (2010) 455-480.

[19] S. De Rosa, F. Franco, T. Polito, Structural similitudes for the dynamic response of plats and assemblies of plates, Mechanical Systems and Signal Processing, 25 (2011) 969980.

[20] S. De Rosa, F. Franco, X. Li, T. Polito, A similitude for structural acoustic enclosures, Mechanical Systems and Signal Processing, 30 (2012) 330-342. 
[21] L. Maxit, J.L. Guyader, Extension of SEA model to subsystem with non-uniform modal energy distribution, Journal of Sound and Vibration, 265, 337-358, 2003.

[22] N. Totaro, J.L. Guyader, Extension of the statistical modal energy distribution analysis for estimating energy density in coupled subsystems, Journal of Sound and Vibration, 331 (2012) 264-289.

[23] L. Maxit, K. Ege, N. Totaro, J.L. Guyader, Non-resonant transmission modelling with statistical modal energy distribution analysis, Journal of Sound and Vibration 333 (2014) 499-519.

[24] À. Aragonès, L. Maxit and O. Guasch, A graph theory approach to identify resonant and non-resonant transmission paths in statistical modal energy distribution analysis. Journal of Sound and Vibration 350 (2015) 91-110.

[25] R. Craig, C.J. Chang, Substructure coupling for dynamic analysis and testing, NASA CR-2791, Washington, DC, 1977.

[26] C. Pierre, Mid-frequency dynamics of complex structural system: assessing the state of the art and defining future research directions, Air Force Office of Scientific Research, Arlington, USA, Report 20030115 102, 2002.

[27] E. Balmes, Structural Dynamics Toolbox \& FEM link, User's guide, SDTools, Paris, France, 2011.

[28] B.R. Mace, P. Shorter, Energy flow models from finite element analysis, Journal of Sound and Vibration, 233 (2000) 369-389.

[29] K. Renji, P.S. Nair, S. Narayanan, Non-resonant response using statistical energy analysis, Journal of Sound and Vibration, 241 (2001) 253-270.

[30] R. Craik, Non-resonant sound transmission through double walls using statistical energy analysis, Applied Acoustics, 64 (2003) 325-341.[31] R.H. Lyon, In-plane contribution to structural noise transmission, Noise Control Engineering Journal, 26 (1986) $22-27$.

[32] A.C. Nilsson, Attenuation of structure-borne sound in super structures on ships, Journal of Sound and Vibration, 55 (1977) 71-91.

[33] N. Kessissoglou, Power transmission in L-shaped plates including flexural and inplane vibration, Journal of the Acoustical Society of America, 115 (2004) 1157-1169. 


\section{FIGURE CAPTIONS}

Figure 1. Geometry of the benchmark structure.

Figure 2. Sub-structuring of the benchmark structure (a) DMF in [7] (b) CB. (Section view of the panels).

Figure 3. Illustration of the different modes shapes for the four coupled plates: (a), normal modes with fixed interface; (b), constraint modes; (c), Characteristic Constraint (CC) modes. Test case \#2. (Note that constraint modes correspond to static deflections and therefore have no associated frequency).

Figure 4. Test case \# 2. (a), Modal energy distribution of subsystem 3. Cross: standard CB results including non-resonant subsystem modes; Square: standard CB results with only resonant subsystem modes. Vertical dash lines indicate the bandwidth of the $1 \mathrm{kHz}$ octave band; (b), $\mathbf{m}_{\phi \psi}^{3} \Xi$, coupling matrix between the $\mathrm{CC}$ modes and the normal modes of subsystem 3.

Figure 5. Modal energy distributions in test case \# 2 for: (a), subsystem 2; (b), subsystem 3. Cross: standard $\mathrm{CB}$ results considering non-resonant modes (reference); circle: reformulated CB results with only resonant subsystem modes and resonant $\mathrm{C}$ modes. Vertical dash lines indicate the bandwidth of the $1 \mathrm{kHz}$ octave band.

Figure 6. Modal energy distributions in test case \# 2 for: (a) subsystem 2; (b) subsystem 3. Cross: original $\mathrm{CB}$ results considering non-resonant modes (reference); circle: $\mathrm{CB}$ results with only resonant subsystem modes and the selected $\mathrm{C}$ modes. Vertical dash lines indicate the bandwidth of the $1 \mathrm{kHz}$ octave band.

Figure 7. Schematic representation of the modal coupling scheme related to the reformulated $\mathrm{CB}$ method $(\mathrm{R}=$ resonant, $\mathrm{NR}=$ non-resonant $)$. 


\section{TABLE CAPTIONS}

Table 1: Subsystem energies and different contributions resulting from the standard CB

method ( $\mathrm{dB}$, ref. $10^{-12} \mathrm{~J},(-)=$ negative contributions). Results for the octave band $1 \mathrm{kHz}$.

Table 2: Number of resonant modes and modal overlap factor for each panel

Table 3: Same type of results than in Table 1 with the standard CB method but only considering the Resonant $(\mathrm{R})$ normal modes. Results for the octave band $1 \mathrm{kHz}$.

Table 4: Same type of results than in Table 1 with the reformulated CB calculation.

Table 5. Subsystem energy results for the reformulated CB calculation with various sets of $\mathrm{C}$ modes. Test case \#2. 


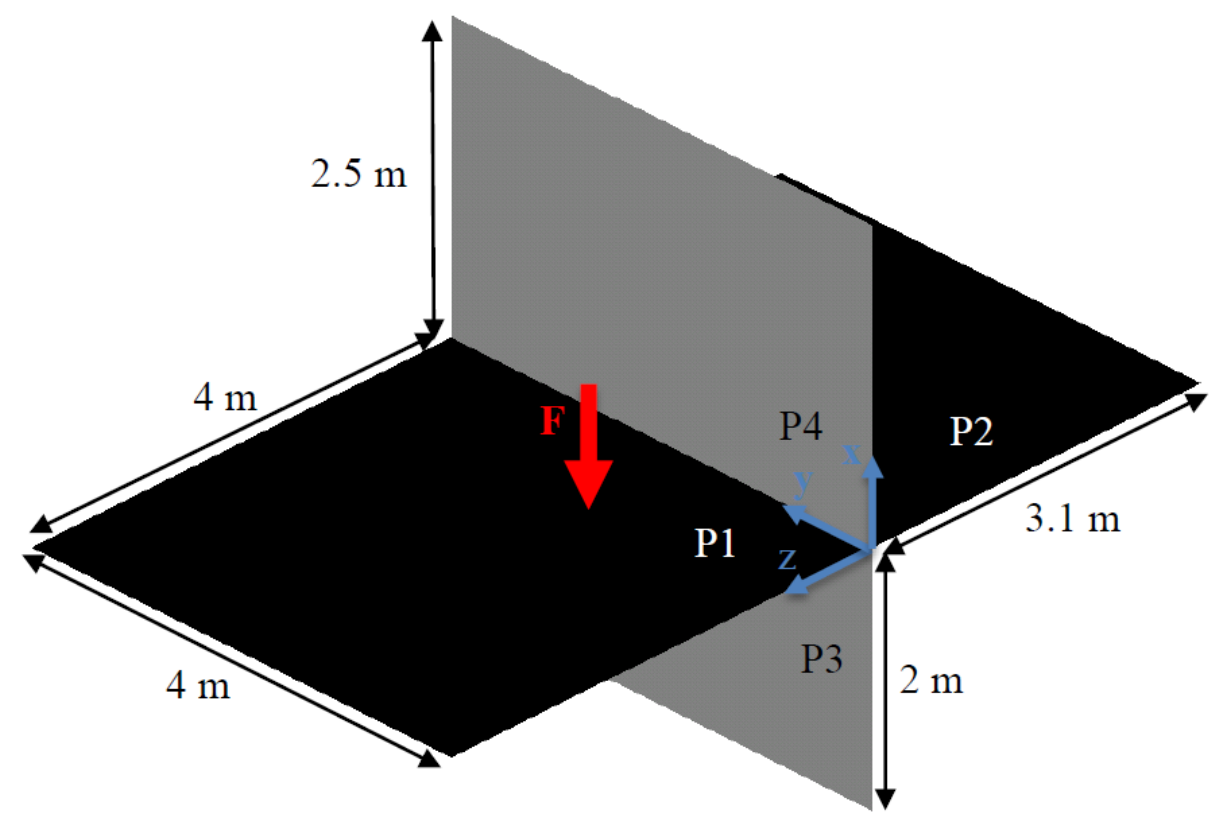

Figure 1. Geometry of the benchmark structure. 


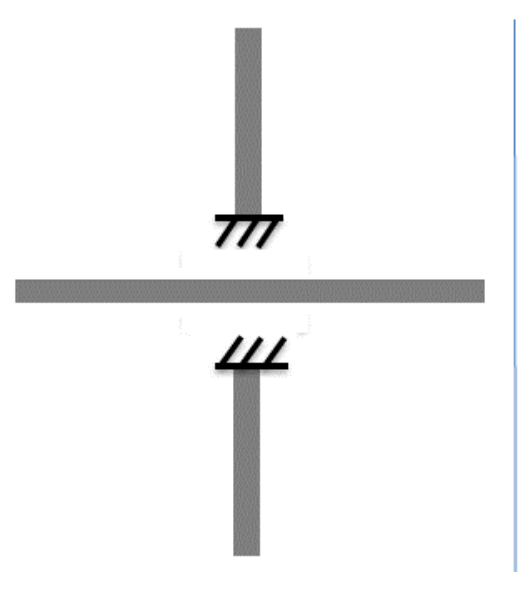

(a)

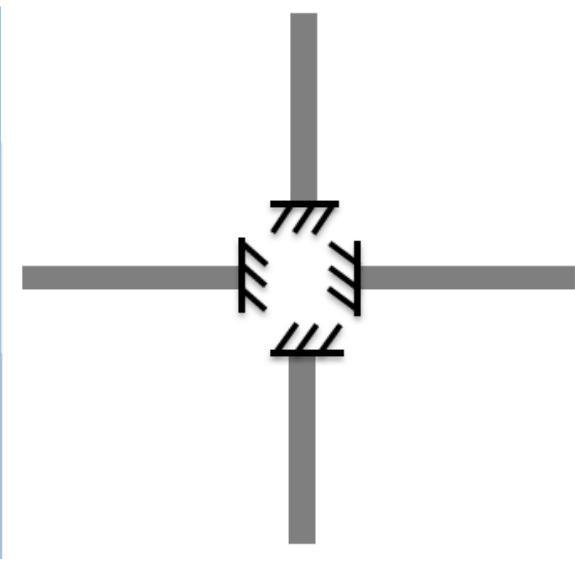

(b)

Figure 2. Sub-structuring of the benchmark structure (a) DMF in [7] (b) CB. (Section view of the panels). 

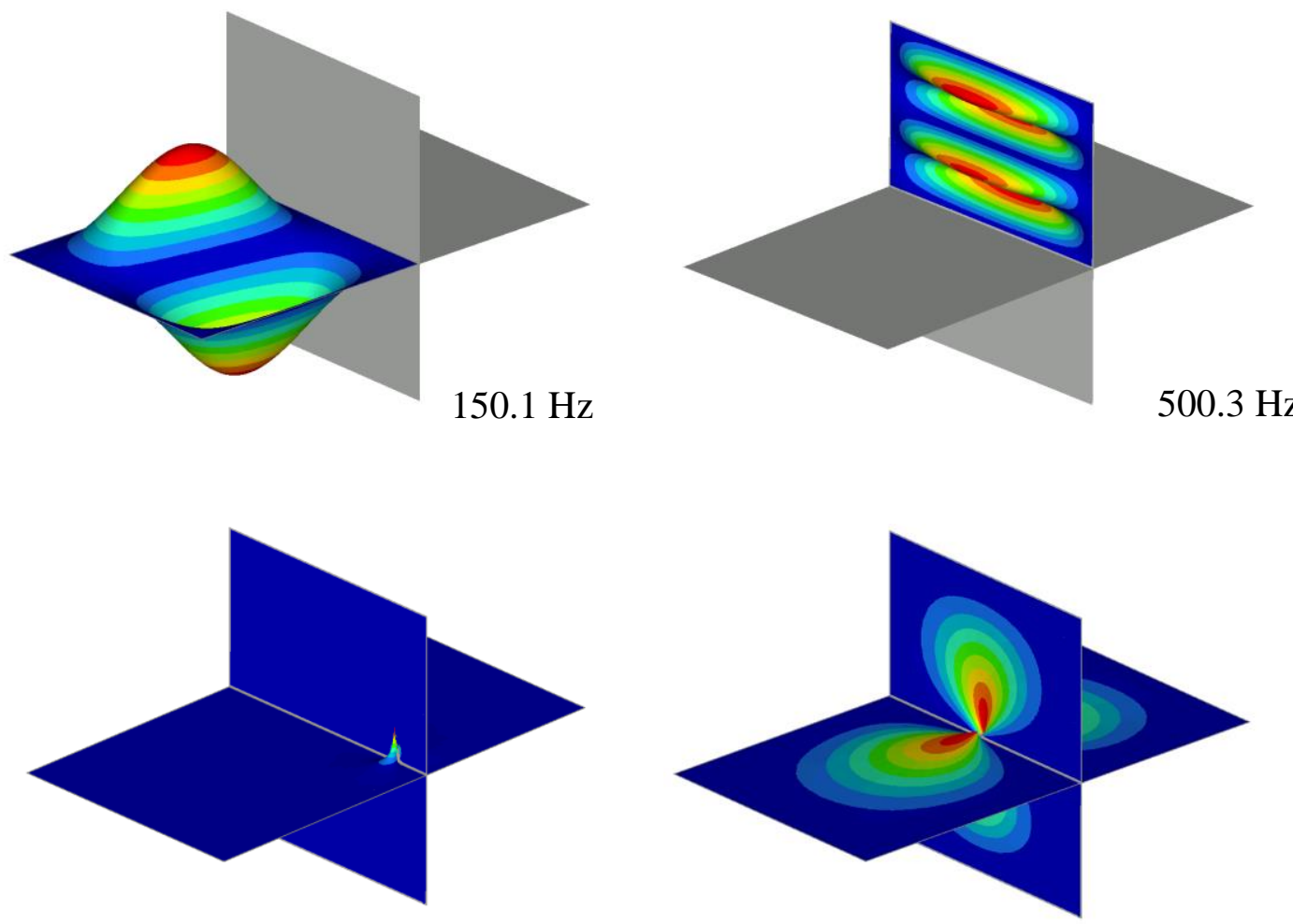

(a)

(b)
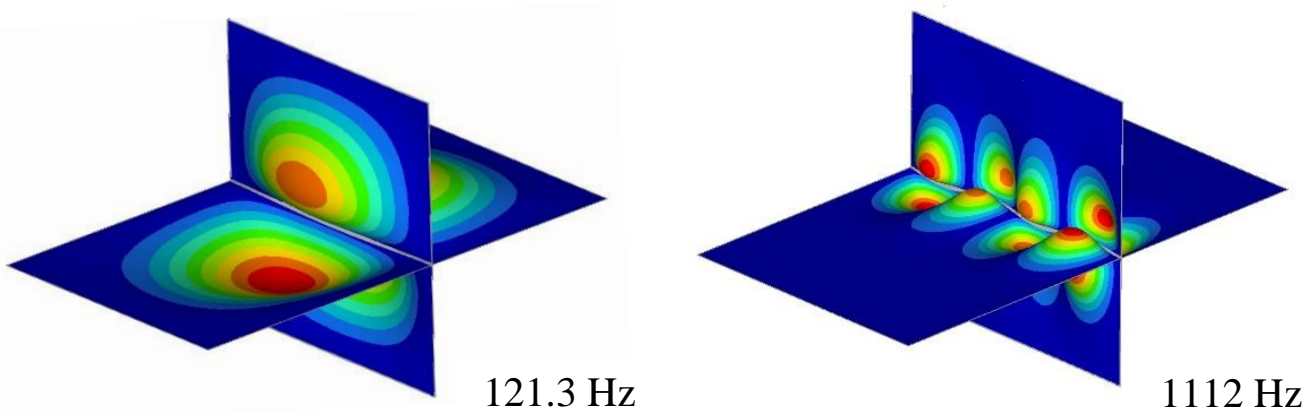

(c)

Figure 3. Illustration of the different modes shapes for the four coupled plates: (a), normal modes with fixed interface; (b), constraint modes; (c), Characteristic Constraint (CC) modes. Test case \#2. (Note that constraint modes correspond to static deflections and therefore have no associated frequency). 


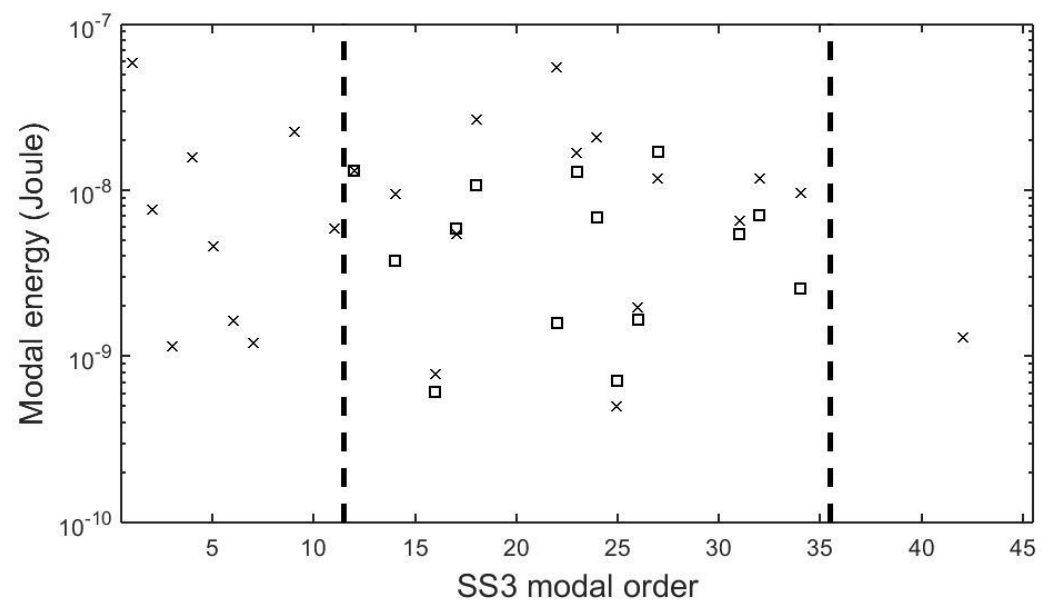

(a)

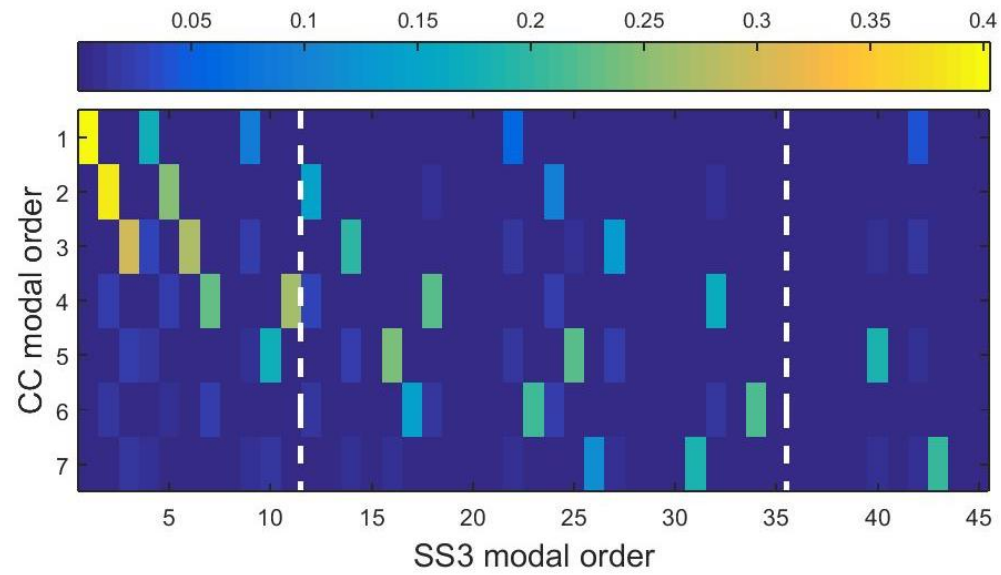

(b)

Figure 4. Test case \# 2. (a), Modal energy distribution of subsystem 3. Cross: standard CB results including non-resonant subsystem modes; Square: standard CB results with only resonant subsystem modes. Vertical dash lines indicate the bandwidth of the $1 \mathrm{kHz}$ octave band; (b), $\mathbf{m}_{\phi \mu}^{3} \Xi$, coupling matrix between the CC modes and the normal modes of subsystem 3 . 


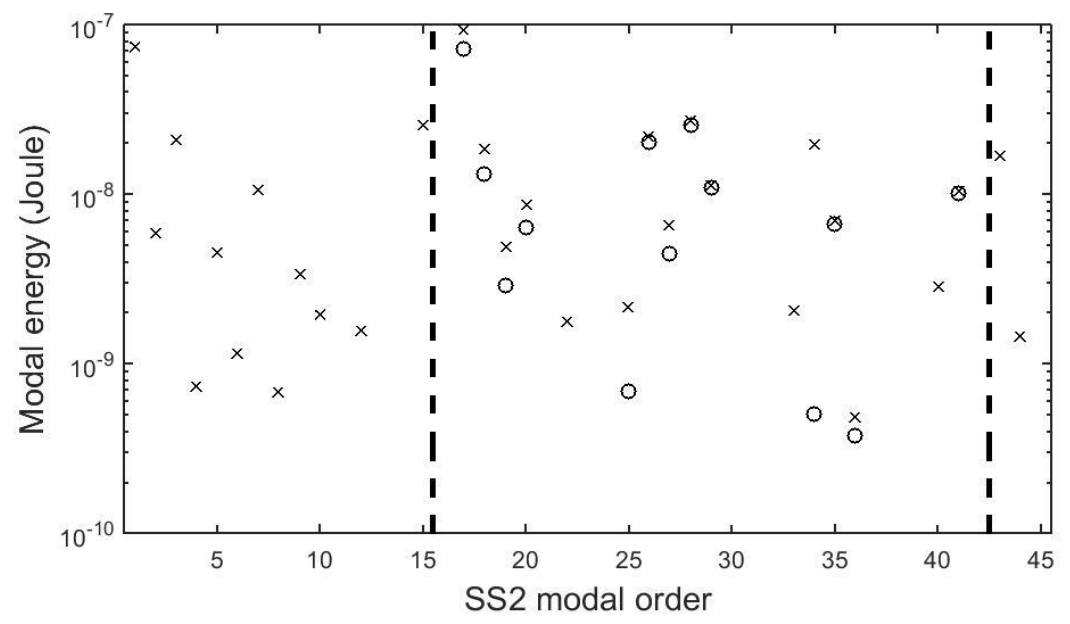

(a)

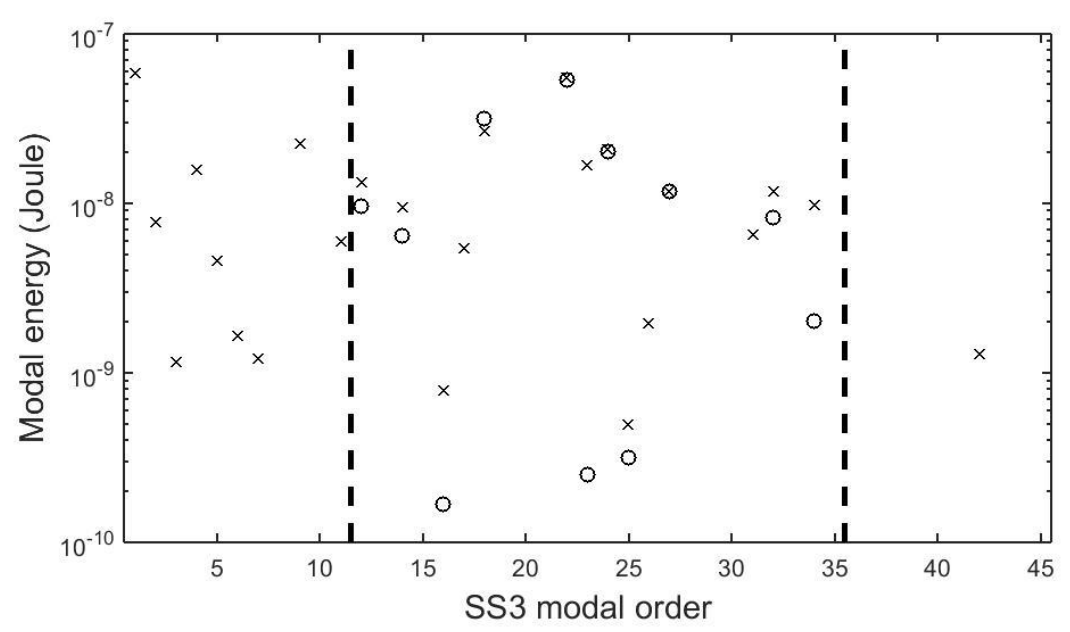

(b)

Figure 5. Modal energy distributions in test case \# 2 for: (a), subsystem 2; (b), subsystem 3. Cross: standard $\mathrm{CB}$ results considering non-resonant modes (reference); circle: reformulated $\mathrm{CB}$ results with only resonant subsystem modes and resonant $\mathrm{C}$ modes. Vertical dash lines indicate the bandwidth of the $1 \mathrm{kHz}$ octave band. 


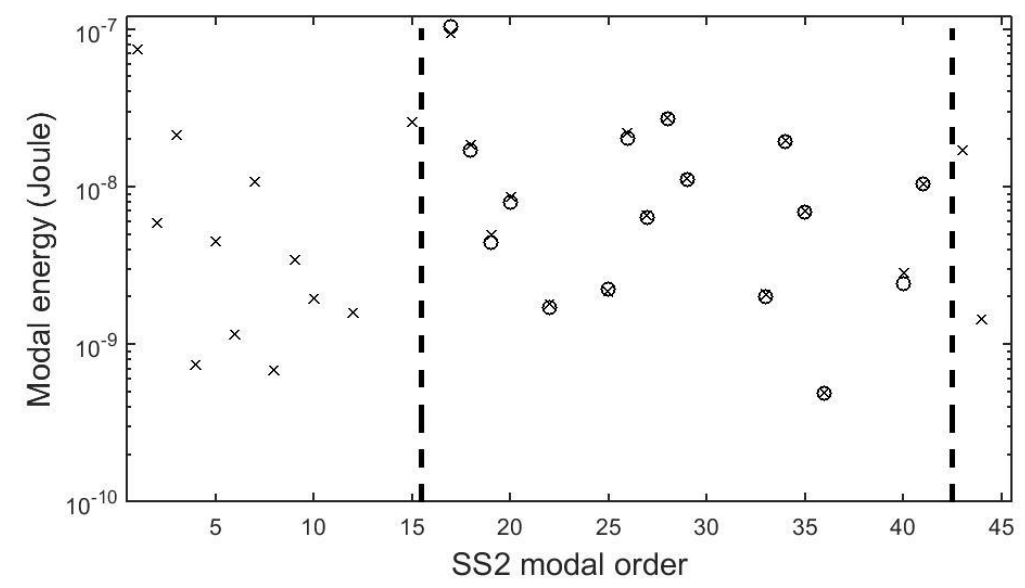

(a)

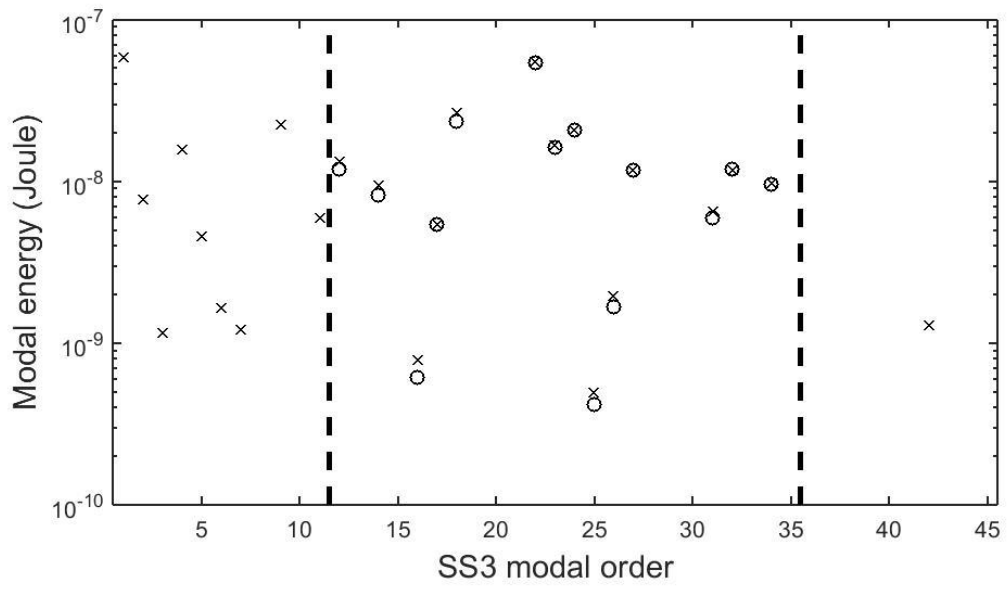

(b)

Figure 6. Modal energy distributions in test case \# 2 for: (a) subsystem 2; (b) subsystem 3. Cross: original $\mathrm{CB}$ results considering non-resonant modes (reference); circle: $\mathrm{CB}$ results with only resonant subsystem modes and the selected $\mathrm{C}$ modes.

Vertical dash lines indicate the bandwidth of the $1 \mathrm{kHz}$ octave band. 


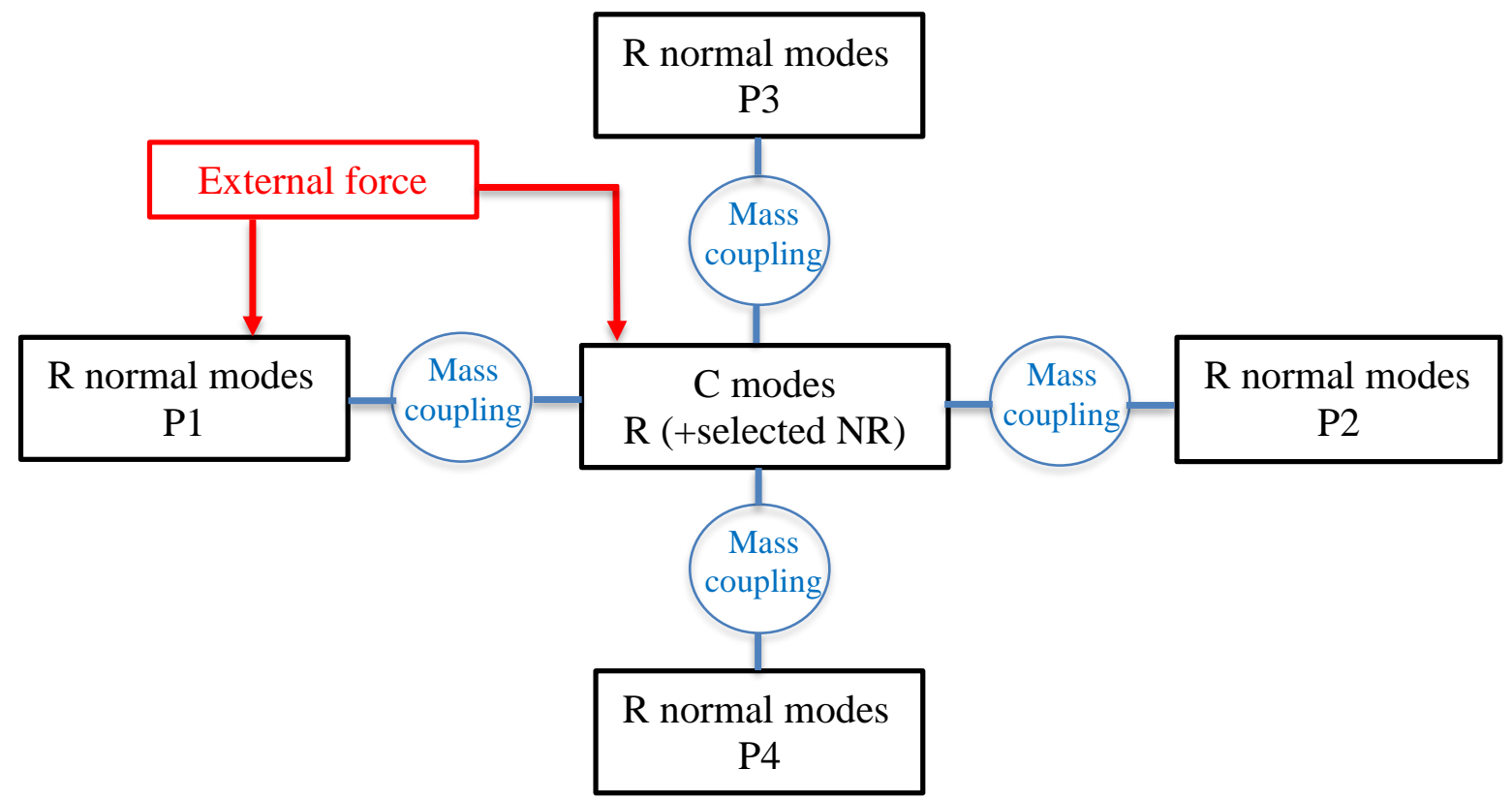

Figure 7. Schematic representation of the modal coupling scheme related to the reformulated $\mathrm{CB}$ method $(\mathrm{R}=$ resonant, $\mathrm{NR}=$ non-resonant $)$. 